\title{
ERK-Mediated Mechanochemical Waves Direct Collective Cell Polarization
}

\section{$\operatorname{AUTHOR}(\mathrm{S}):$}

Hino, Naoya; Rossetti, Leone; Marín-Llauradó, Ariadna; Aoki, Kazuhiro; Trepat, Xavier; Matsuda, Michiyuki; Hirashima, Tsuyoshi

\section{CITATION:}

Hino, Naoya ...[et al]. ERK-Mediated Mechanochemical Waves Direct Collective Cell Polarization. Developmental Cell 2020, 53(8): 646-660.e8

\section{ISSUE DATE:}

2020-06-22

URL:

http://hdl.handle.net/2433/253559

\section{RIGHT:}

(c) 2020. This manuscript version is made available under the CC-BY-NC-ND 4.0 license

http://creativecommons.org/licenses/by-nc-nd/4.0/; The full-text file will be made open to the public on 22 JUNE 202 in accordance with publisher's 'Terms and Conditions for Self-Archiving'; This is not the published version. Please cite only the published version.; この論文は出版社版でありません。引用の際には出版社版をご確認ご利用ください。 


\section{ERK-mediated mechanochemical waves direct collective cell polarization}

Naoya Hino ${ }^{1}$, Leone Rossetti ${ }^{2}$, Ariadna Marín-Llauradó ${ }^{2}$, Kazuhiro Aoki $^{3,4,5}$, Xavier Trepat ${ }^{2,6,7,8}$,

4 Michiyuki Matsuda $^{1,9^{*}}$, Tsuyoshi Hirashima ${ }^{9,10,11^{*}}$

5

6 Laboratory of Bioimaging and Cell Signaling, Graduate School of Biostudies, Kyoto

7 University, Sakyo-ku, Kyoto 606-8501, Japan

$8 \quad{ }^{2}$ Institute for Bioengineering of Catalonia, Barcelona 08028, Spain

$9{ }^{3}$ Quantitative Biology Research Group, Exploratory Research Center on Life and Living

10 Systems (ExCELLS), National Institutes of Natural Sciences, 5-1 Higashiyama, Myodaiji-cho,

11 Okazaki, Aichi 444-8787, Japan.

$12{ }^{4}$ Division of Quantitative Biology, National Institute for Basic Biology, National Institutes of

13 Natural Sciences, 5-1 Higashiyama, Myodaiji-cho, Okazaki, Aichi 444-8787, Japan.

145 Department of Basic Biology, School of Life Science, SOKENDAI (The Graduate University for Advanced Studies), 5-1 Higashiyama, Myodaiji-cho, Okazaki, Aichi 444-8787, Japan.

${ }^{6}$ Faculty of Medicine, University of Barcelona, Barcelona, Spain

${ }^{7}$ Institució Catalana de Recerca i Estudis Avançats (ICREA), Barcelona, Spain

$18{ }^{8}$ Center for Networked Biomedical Research on Bioengineering, Biomaterials and 19 Nanomedicine (CIBER-BBN), Barcelona, Spain.

$20{ }^{9}$ Department of Pathology and Biology of Diseases, Graduate School of Medicine, Kyoto

21 University, Sakyo-ku, Kyoto 606-8501, Japan

$22{ }^{10}$ Japan Science and Technology Agency, PRESTO, Sakyo-ku, Kyoto 606-8501, Japan

$23{ }^{11}$ Lead Contact

\section{${ }^{*}$ Corresponding Authors:}

matsuda.michiyuki.2c@kyoto-u.ac.jp (M.M.), hirashima.tsuyoshi.2m@kyoto-u.ac.jp (T.H.)

\section{Lead Contact:}

Tsuyoshi Hirashima, hirashima.tsuyoshi2m@kyoto-u.ac.jp 


\section{Summary}

32 During collective migration of epithelial cells, the migration direction is aligned over a tissue33 scale expanse. Although the collective cell migration is known to be directed by mechanical 34 forces transmitted via cell-cell junctions, it remains elusive how the intercellular force 35 transmission is coordinated with intracellular biochemical signaling to achieve collective 36 movements. Here we show that intercellular coupling of ERK-mediated mechanochemical 37 feedback yields long-distance transmission of guidance cues. Mechanical stretch activates ERK 38 through EGFR activation, and ERK activation triggers cell contraction. The contraction of the 39 activated cell pulls neighboring cells, evoking another round of ERK activation and contraction 40 in the neighbors. Furthermore, anisotropic contraction based on front-rear polarization 41 guarantees unidirectional propagation of ERK activation, and in turn, the ERK activation waves 42 direct multicellular alignment of the polarity, leading to long-range ordered migration. Our 43 findings reveal that mechanical forces mediate intercellular signaling underlying sustained 44 transmission of guidance cues for collective cell migration.

\section{Keywords}

48 Collective cell migration; ERK/MAPK; EGFR; FRET; front-rear polarity; intercellular signal 49 transfer; mathematical model; mechanochemical feedback; mechanotransduction; wave 50 propagation. 


\section{Introduction}

Collective cell migration underpins various fundamental biological processes, including embryonic development and tissue repair (Friedl and Gilmour, 2009; Mayor and EtienneManneville, 2016). A migrating cell cluster exhibits multicellular coordination of cellular parameters, such as cytoskeleton organization (Farooqui and Fenteany, 2005), organelle positioning (Carvalho et al., 2019; Farooqui and Fenteany, 2005; Reffay et al., 2011), and cell velocity (Petitjean et al., 2010; Tlili et al., 2018). Directionality of these parameters in each cell can be provided by two mechanisms. First, cells sense a direction from global external cues, such as a gradient of chemoattractant or substrate stiffness (Haeger et al., 2015; Shellard and Mayor, 2019). Second, directional cues are transmitted from a leading edge of a cell cluster to the bulk; cells located at the edge, referred to as leader cells, sense the microenvironment to spread out and dictate the direction of follower cells located behind the leader cells (Omelchenko et al., 2003; Reffay et al., 2014; Yamaguchi et al., 2015). In the latter case, all follower cells as well as the leader cells generate mechanical forces to actively migrate, and those forces are orchestrated as cooperative intercellular forces over the cell cluster (SerraPicamal et al., 2012; Tambe et al., 2011; Trepat et al., 2009). It has also been shown that the mechanical forces transmitted via cell-cell junctions provide local cues to direct an ordered cell migration in a cluster (Das et al., 2015; Tambe et al., 2011); however, our understanding of the signaling molecules responsible for the long-range transmission of the mechanical forces is far from complete.

Extracellular signal-regulated kinase (ERK), a serine/threonine kinase, plays critical roles in mechanotransduction that regulates differentiation (Tanabe et al., 2004), epithelial cell division (Gudipaty et al., 2017), and tissue homeostasis (Moreno et al., 2019). Earlier studies have shown that ERK activation propagates as multiple waves from leader cells to the follower cells during collective cell migration using in vitro cultured cells (Handly et al., 2015; Matsubayashi et al., 2004; Nikolic et al., 2006) and in vivo mouse ear skin (Hiratsuka et al., 2015). Recently, we have demonstrated that the ERK activation waves orient the directed migration of a cell cluster against the wave direction, indicating a critical role of the ERK activation waves in coordinated cell migration (Aoki et al., 2017). However, it remains largely unknown how cells harness the mechanotransduction and the ERK activation waves to coordinate collective cell migration.

In this study, by combining Förster resonance energy transfer (FRET)-based biosensors (Harvey et al., 2008; Komatsu et al., 2011), an optogenetic tool (Aoki et al., 2013), traction 
force microscopy (Trepat et al., 2009), and mathematical modeling, we show that each follower cell possesses a mechanochemical feedback system, in which stretch-induced ERK activation triggers cell contraction. Intercellular coupling of the ERK-mediated mechanochemical feedback enables sustained propagation of ERK activation and contractile force generation, leading to multicellular alignment of front-rear cell polarity over long distances. Thus, our study clarifies a mechanism of intercellular communication underlying long-range sustained transmission of directional cues for collective cell migration.

\section{Results}

\section{Cell deformation waves precede ERK activation waves}

To investigate the relationship between ERK activation and cell deformation during collective cell migration, Madin-Darby canine kidney (MDCK) cells confluently seeded within compartments of a silicone confinement were released for collective cell migration. ERK activity and cell deformation were evaluated by FRET imaging with EKAREV-NLS, by which ERK activation is quantified as an increase in the FRET/CFP ratio (Komatsu et al., 2011), and particle image velocimetry (PIV)-based image processing, respectively. As the cells migrated toward free spaces, sustained ERK activation waves were propagated from the leader cells to the follower cells (Figures 1A and 1B; Movie S1), in agreement with previous reports (Aoki et al., 2017; Matsubayashi et al., 2004). The cell deformation, i.e., extension and shrinkage, was quantified by the cell strain rate in the direction of collective cell migration ( $\mathrm{x}$-strain rate). Similar to the ERK activity, the $\mathrm{x}$-strain rate exhibited repeated positive and negative values, namely extension and shrinkage, which propagated from the leader cells to the follower cells (Figures 1A and 1C; Movie S1), as described previously (Serra-Picamal et al., 2012; Tlili et al., 2018). Hereinafter, we refer to the propagation of the $\mathrm{x}$-strain rate as cell deformation waves (Rodriguez-Franco et al., 2017).

To analyze the correlation between the ERK activation and the cell deformation, timeseries data of the ERK activity and those of the $\mathrm{x}$-strain rate were collected at the single cell level. We then calculated ERK activation rate, i.e., the ERK activity change per min, to compare not ERK activity itself but a time derivative of ERK activity and $\mathrm{x}$-strain rate. We noticed that the ERK activation rate and the $\mathrm{x}$-strain rate oscillated almost synchronously with an approximately 90 min period (Figure 1D). Furthermore, temporal cross-correlation analysis revealed that the ERK activation rate lagged 3 min behind the $\mathrm{x}$-strain rate (Figure 1E), 
118 indicating that the cell deformation waves precede the ERK activation waves. In other words,

119 cells are first extended, followed by ERK activation, and then the cells start contracting.

Cell extension triggers ERK activation via EGFR signaling

122 Because the cell extension precedes ERK activation, we reasoned that mechanical stretch activates ERK during collective cell migration. To demonstrate this, we stretched MDCK cells plated on an elastic silicone plate and compared the ERK activity before and after the mechanical stretch. As anticipated, uniaxial 50\% stretch of the MDCK cells resulted in ERK activation (Figures 2A and 2B), indicating that passive extension of cells activates ERK.

127 Furthermore, the ERK activation almost linearly responded to different degrees of stretch, and $30 \%$ stretch was sufficient for triggering significant ERK activation, indicating that ERK activation scales with degrees of stretch.

Previously, we reported that EGFR and a disintegrin and metalloprotease 17

131 (ADAM17), which catalyzes the shedding of membrane-tethered EGFR ligands (Maretzky et 132 al., 2011a), affect ERK activation waves (Aoki et al., 2017; Aoki et al., 2013). Consistent with 133 those reports, either treatment with an inhibitor of EGFR, PD153035, or that of matrix 134 metalloproteinases (MMPs) and ADAMs, marimastat, suppressed the ERK activation waves 135 during collective cell migration (Figure S1). These observations strongly suggest that the 136 stretch-induced ERK activation requires EGFR and ADAM17 activity. In fact, both PD153035 137 and marimastat suppressed the stretch-induced ERK activation (Figure 2C). Remarkably, 138 immunoblotting showed that cell stretch transiently increased auto-phosphorylated EGFR, 139 followed by phosphorylation of the downstream kinases including RAF1, MEK1/2, and 140 ERK1/2 (Figures 2D-2H). Together, these results indicate that cell stretch activates EGFR and 141 its downstream signaling molecules, including ERK. ERK activation induces cell contraction via Rho-associated kinase activation

144 Because the ERK activation precedes cell shrinkage (Figure 1E), we speculated that ERK activation would induce cell contraction. To prove this hypothesis, we used 2paRAF, a lightinducible ERK activation system based on cryptochrome 2 (CRY2)-CIBN dimerization (Aoki et al., 2013; Kennedy et al., 2010; Kinjo et al., 2019). In this system, RAF1 fused with CRY2

148 is recruited to the plasma membrane by heterodimerization of CRY2 and membrane-anchored 149 CIBN upon blue light exposure, culminating in ERK activation. We seeded cells with and without 2paRAF (2paCRY2-RAF1/CIBN-mScarlet-I-CAAX) expression into two separated 
151 compartments of a silicone confinement, respectively, and formed an interface of the two cell

152 populations by the removal of the confinement (Figure 3A). Upon ERK activation by blue light

153 exposure, the interface shifted toward the side of the cells expressing 2paRAF (Figures 3B and

154 3C; Movie S2). Moreover, inhibiting the ERK activation with a MAPK/ERK kinase (MEK)

155 inhibitor, trametinib, treatment suppressed the interface shift (Figures 3B and 3C; Movie S2).

156 Furthermore, even with the inhibition of the ERK activity propagation in the cells without

157 2paRAF expression by treating an inhibitor of EGFR PD153035, a degree of the light-induced

158 contraction of the cells expressing 2paRAF was not significantly different from a case without

159 the inhibitor (Figures S2A and S2B), indicating that the cell contraction is due to the optogenetic

160 ERK activation but not to the ERK activation in the neighbors. Thus, these results clearly

161 indicate that the ERK activation induces cell contraction of confluent MDCK cells. To confirm

162 ERK activation also triggers cell contraction during collective cell migration, we tested the

163

164

165

166

167

168

169

170

171

172

173

174

175

176

177

178

179

180

181

182

183 effect of ERK inhibition either with an inhibitor of EGFR, PD153035, or that of MAPK/ERK kinase (MEK), trametinib, on deformation of migrating cells. Both PD153035 and trametinib treatment damped the oscillation of the $\mathrm{x}$-strain rate (Figure S2C-S2F). Thus, ERK activation is required for cell deformation during collective cell migration.

Cell contraction is driven by actomyosin, which involves Rho-associated kinase (ROCK)-mediated regulation (Amano et al., 1996; Kimura et al., 1996; Totsukawa et al., 2000). Thus, we examined the effects of a ROCK inhibitor, Y-27632, on the ERK-induced cell contraction. We found that Y-27632 suppressed the ERK-induced cell contraction (Figure 3B and $3 \mathrm{C}$; Movie S2). This observation prompted us to examine whether ERK induces ROCK activation. To this end, we used a cytosolic FRET biosensor for ROCK activity (Li et al., 2017). After optogenetic ERK activation, ROCK activity increased, then plateaued within 12 min after the blue light exposure (Figures 3D and 3E). We next examined the ROCK activity dynamics during collective cell migration. Interestingly, ROCK activity exhibited repeated unidirectional wave propagation from leader cells to the follower cells, as did the ERK activity (Figures $3 \mathrm{~F}$ and 3G; Movie S3). Inhibition of ERK with trametinib abolished the propagation of ROCK activation waves (Figure 3H; Movie S3) as well as temporal oscillatory activation in each cell (Figure 3I). In stark contrast, inhibition of ROCK with Y-27632 abolished the oscillatory activity in each cell and also decreased the basal activity of ROCK drastically (Figures 3J and 3K; Movie S3), indicating that ERK activity is responsible only for the oscillatory component of ROCK activity. Thus, we conclude that ROCK is activated downstream of ERK and is integral to ERK-induced cell contraction. 


\section{ERK activation decreases traction forces and accumulates $\mathbf{F}$-actin at the cell-cell}

186

187

188

189

190

191

192

193

194

195

196

197

198

199

200

201

202

203

204

205

206

207

208

209

210

211

212

213

214

215

216

\section{interface}

How does ERK activation alter mechanical force generation to induce contraction? To answer this question, we combined light-inducible ERK activation with traction force microscopy (Trepat et al., 2009), which allows the measurement of traction forces loaded by cells on the substrate. On a substrate of polyacrylamide gel embedded with fluorescent beads, the interface between the cells with and without 2paRAF expression was formed, as already described (Figure 3A). The traction force exerted by the 2paRAF-expressing cells markedly decreased within 40 min after the start of blue light exposure (Figures 3L and 3M; Movie S4). By contrast, inhibiting ERK with trametinib restored the traction force generation (Figure 3N), confirming that ERK activation suppresses force loading on the substrate. The gradual increase in traction force by the cells without 2paRAF expression in Figure $3 \mathrm{M}$ and $3 \mathrm{~N}$ is independent of the optogenetic ERK activation in the neighboring 2paRAF-expressing cell cluster because the traction force increased over time even without blue light exposure (Figure S2G and S2H). This is likely because increasing cell density by proliferation downregulates ERK activity (Aoki et al., 2013). Moreover, we found that subcellular localization of F-actin in migrating cells is altered depending on the ERK activity level (Figures $3 \mathrm{O}$ and 3P). ERK activation by epidermal growth factor (EGF) promoted F-actin localization at the lateral side of the cells. By contrast, ERK inhibition by trametinib treatment produced stress fibers at the basal side of the cells. Therefore, these results suggest that ERK activation triggers cell contraction by accumulating F-actin at the cell-cell interface and triggering predominant force loading on the interface while suppressing traction force generation.

\section{Zonal cell contraction initiates sustained unidirectional ERK activation waves at the}

\section{interface}

We have shown that each cell possesses an ERK-mediated mechanochemical feedback system coupling cell deformation and ERK activation: ERK is activated by cell extension, and the activated ERK induces cell contraction. Considering the tight physical connection between epithelial cells, contraction of a cell cluster should stretch cells in the adjacent cluster, mainly at its border, thereby generating the ERK activity propagation. To test this hypothesis, we used a rapamycin-activatable (RA) Rho guanine nucleotide exchange factor (GEF) system to induce contraction of a cell cluster (Inoue et al., 2005; van Unen et al., 2015). We first seeded cells 
217 carrying the RA Rho GEF expression system into a confinement (Figure 4A), and then seeded 218 EKAREV-NLS cells after the removal of the confinement, resulting in the formation of an 219 interface between cells expressing RA Rho GEF and cells expressing EKAREV-NLS. Before 220 rapamycin treatment, ERK activity was randomly propagated without any preferential direction 221 (Figure 4B; Movie S5). Upon rapamycin treatment, the cells with RA Rho GEF began to contract, resulting in the extension of the adjacent EKAREV-NLS-expressing cell cluster toward the RA Rho GEF-expressing cell clusters (Figures 4C and 4D; Movie S5). With this trigger, ERK activation waves emerged at the interface and were unidirectionally propagated toward the extended EKAREV-NLS-expressing cell cluster (Figures 4C-4E). In addition, the displacement of the EKAREV-NLS-expressing cells was oriented toward the RA Rho GEFexpressing cell clusters and was directed opposite the ERK activation waves (Figure 4F). Thus, these results suggest that cell contraction generates ERK activation waves in the adjacent cell cluster.

We then asked whether a cell-cell tight connection is required for intercellular propagation of ERK activation waves. To test this, we knocked-out $\alpha$-catenin, a major cell-cell junction component (Nagafuchi et al., 1994), in MDCK cells (Figure 4G). As expected, E-cadherin at the cell-cell junction was reduced in the $\alpha$-catenin $\mathrm{KO}$ cells in comparison with wild-type (WT) cells (Figure 4H). Importantly, there was no significant difference in the sensitivity of the WT and $\alpha$-catenin KO cells to EGFR stimulation by EGF (Figure 4I). Thus, the cells possess equal sensitivity to EGFR stimulation even in $\alpha$-catenin KO. Nevertheless, coordinated ERK activity propagation was severely disrupted in the $\alpha$-catenin KO cells (Figures 4J and 4K; Movie S6), indicating that intercellular mechanical linkage is required for ERK activity propagation. Thus, we conclude that intercellular force transmission mediates ERK activation waves.

\section{Front-rear cell polarization is required for the unidirectional propagation of ERK}

243 We next investigated how unidirectional propagation of ERK activation waves is achieved 244 during collective cell migration. If cells contract isotropically, the loss of directional information would impede the unidirectional propagation of ERK activation waves. Therefore, there must be a mechanism by which the directional information is retained during the wave propagation. We found that the cell deformed preferentially along the direction of migration (Figures 5A-5C), to an extent sufficient for activating ERK (Fig. 2B), indicating that cells 
250 light chain, a marker of contractile myosin, and F-actin accumulated at the basal rear side of 251 migrating cells (Figures 5D, S3A, and S3B), which are indicative of polarized rear contraction. 252 Some cells showing the dense actomyosin fibers elongate almost perpendicular to the direction 253 of cell migration. This suggests that cell contraction toward the migration direction would cause 254 slight extension perpendicular to the direction of migration because the cell volume does not 255 largely change during the cell contraction. The presence of a front-rear cell polarity was also 256 confirmed by the localization of GM130, a Golgi apparatus marker, known to be located in 257 front of the nucleus in some types of migrating cells (Magdalena et al., 2003; Nobes and Hall, 258 1999). When cells were examined immediately after release from confinement ( $0 \mathrm{~h}$ migration), 259 the Golgi orientation relative to the nucleus was not biased toward the leading edge (Figures $260 \mathrm{~S} 3 \mathrm{C}$ and S3D). By contrast, $21 \mathrm{~h}$ after the initiation of collective cell migration, the Golgi orientation was biased toward the direction of the migration over more than a $1 \mathrm{~mm}$ range from the leader cells (Figures 5E and 5F). To quantify the degree of alignment, we defined the directedness of the Golgi orientation (Figure 5G), and found that the Golgi directedness first increased around the leader cells, and then the increase spread through the follower cells over time (Figure 5H). In addition, the directedness of ERK activation waves and that of migration showed similar dynamics to the Golgi directedness (Figures 5I and 5J). Thus, these results clarify that alignment of front-rear cell polarity in follower cells positively correlates with unidirectionality of ERK activation waves.

To investigate the requirement of front-rear cell polarization for the unidirectional ERK activation waves, we knocked down Rac1 or Cdc42, which are required for the establishment 271 of front-rear polarization (Nobes and Hall, 1999). Expression of short hairpin RNAs reduced 272 Rac1 and Cdc42 expression to 50\% and 30\%, respectively (Figures S3E and S3F). In the 273 knocked-down cells, the front-rear polarization of Golgi in the leader cells was still directed in 274 the direction of the migration; however, that of the follower cells was impaired after $21 \mathrm{~h}$ migration (Figures 5K and 5L). Moreover, the unidirectionality of the ERK activation waves and the alignment of migration direction were also impaired in the follower cells of the knocked-down cells (Figures 5M and 5N; Movie S7). Taken together, these results demonstrate

278 that the alignment of front-rear polarization toward the direction of the migration is required

279 for the unidirectional ERK activation waves and the ordered cell migration over long distances 280 from the leading edge. 
283

We further addressed the relationship between the ERK activation waves and the front-rear polarization. Previously, it has been proposed that force loading on cell-cell junctions directs front-rear polarization (Das et al., 2015). Consistent with this, disruption of cell-cell junctions by $\alpha$-catenin $\mathrm{KO}$ abolished the alignment of the front-rear polarization toward the direction of migration (Figures S4A-S4C). From this observation, we speculated that ERK-induced contractile force generation would direct the front-rear polarization in neighboring follower cells. As expected, inhibition of ERK activation with trametinib suppressed the alignment of the front-rear polarization and migration directionality in the follower cells at $21 \mathrm{~h}$ after migration (Figures 6A-6C and S4D; Movie S8). Interestingly, constitutive ERK activation by 12-O-tetradecanoylphorbol 13-acetate (TPA) also inhibited the alignment of the front-rear polarization and migration directionality in the follower cells (Figures 6A-6C and S4E; Movie S8), suggesting that periodic ERK activation in the form of waves is important for polarizing follower cells. We then tested whether synthetic ERK activation waves can enhance the directedness of the Golgi polarization in the follower cells. To this end, we used MDCK cells expressing 2paRAF (2paCRY2-RAF1/CIBN-CAAX) and Golgi-7-mCherry as a Golgi apparatus marker. Those cells were confluently seeded in a confinement, and then released for migration with an EGFR inhibitor PD153035 to suppress autonomous ERK activation waves (Figure 6D). Of note, inhibition of EGFR signaling suppresses ERK activation waves in follower cells, but ERK activation in the cells near the leading edge remains (Figure S1B and S1C). Thus, the leader cells still migrate, even with EGFR signaling inhibition, while follower cell migration is abrogated (Aoki et al., 2017). Under this condition, we created optogenetic ERK activation waves by shifting patterned blue light-illumination to mimic spontaneous ERK activation waves; i.e., $\sim 35 \mu \mathrm{m}$ width and $400 \mu \mathrm{m}$ intervals at $3 \mu \mathrm{m} \mathrm{min}^{-1}$ velocity (Figure $6 \mathrm{D}$ ). With the synthetic ERK activation waves, the front-rear cell polarizations were significantly aligned even in follower cells more than $600 \mu \mathrm{m}$ distant from the leader cells, compared with the samples in the absence of ERK activation waves (Figures 6E and 6F; Movie S9). Thus, these results clearly demonstrate that ERK activation waves orient the front-rear polarity in cells against the direction of the waves. Collectively, we conclude that unidirectional ERK activation waves and multicellular alignment of the front-rear polarization propagate cooperatively, enabling long-distance transmission of directional cues for collective cell migration.

\section{Modeling cellular mechanochemical feedback with polarity demonstrates long-range}




\section{unidirectional ERK activation waves}

317 Finally, we developed a mathematical model to understand the role of ERK-mediated 318 mechanochemical waves in collective cell migration. We employed a two-dimensional cellular 319 Potts model (CPM) to represent behavior of the epithelial monolayer at a single cell scale 320 (Glazier and Graner, 1993; Hirashima et al., 2017; Merks and Glazier, 2005). In this model, each cell morphology is represented as a cluster of regular lattices, and a state of lattices determine an energy of the system. The dynamics of a multicellular system proceeds stochastically on the basis of an energy minimization using a Monte Carlo simulation algorithm. We here regard a unit of trials in Monte Carlo simulation (Monte Carlo steps: MCS) as $1 \mathrm{~h}$ in experiments. The energy in the model includes essential properties in multicellular mechanics, i.e., intercellular adhesion, cell elasticity, and active cellular contraction (Guillot and Lecuit, 2013; Heisenberg and Bellaiche, 2013). In addition, the cells possess an inherent orientation regarding the polarized rear contraction, equivalent to the front-rear polarity defined as the Golgi orientation in our experiments (Figure 7A). In our model, orientations of the cell polarity in individual cells tend to be aligned as a result of the physical interaction with neighboring cells (Supplementary notes) (Hirashima et al., 2013; Peyret et al., 2019; Szabo et al., 2010). The anisotropy along the front-rear polarity and the strength of cell contraction are governed by the cell polarity parameter $\omega$ and the ERK activity, respectively. That is, the cell contraction is isotropic when $\omega=0$ and polarized for the rear side of the cell with increasing $\omega$ (Figure 7B); we set $\omega=1$ as suggested in experiments (Figures 5A-5J), unless otherwise noted. In simulations, we assumed that the follower cells obey the stretch-induced contraction, while the leader cells keep migrating towards the free space.

We performed simulation analysis under a similar condition to the confinement release assay, and confirmed that the unidirectional ERK activation waves from the leader cells to the follower cells were reproduced (Figures 7C and 7D; Movie S10). Moreover, the simulation results well mimicked experimental measurements in terms of spatio-temporal profiles of frontrear polarity directedness, ERK wave directedness, and migration directedness (Figure 7E). We then examined the effect of the cell polarity level $\omega$ on the directedness. For any type of directedness, the spatial range with high values becomes more limited around the leading cells, with the decrease of $\omega$ (Figure 7F; Movie S10) as observed with disruption of polarity proteins (Figure $5 \mathrm{~K}-5 \mathrm{~N}$ ). This indicates that the cell anisotropy in contraction along the front-rear polarity is essential for long-range multicellular guidance. In addition, we investigated the effect of ERK activation waves on the polarity directedness (Figures $7 \mathrm{G}$ and $7 \mathrm{H}$ ). Even without the 
349 ERK activation waves, the front-rear polarity in the follower cells around the leading edges is 350 relatively directed toward the front because the migrating leader cells pull near-follower cells; 351 however, the polarity directedness gradually decreases with distance from the leader cells. By 352 contrast, the polarity directedness is maintained long-range from the leader cells via the ERK 353 activation waves (Figure 7H). Hence, ERK activation waves direct the front-rear polarity of the 354 follower cells, consistent with the experiments with optogenetic ERK activation waves (Figure $3556 \mathrm{~F})$.

356 Further in silico analysis led to the finding that the strength of cell-cell adhesion $\gamma$ 357 controls synchronization of the ERK activity along a perpendicular axis to its propagation 358 direction (y-axis in Figure 7I). We introduced a spatial correlation length of the ERK activity 359 along the $y$-axis as a measure of its synchronization, and examined a dependency of $\gamma$ on the spatial correlation length. Our numerical investigation clearly shows that the spatial correlation length increases with increasing cell-cell adhesion strength $\gamma$ (Figure 7J), indicating that multicellular integrity via intercellular mechanical linkages plays a key role in the ERK activity synchronization. Taken together, tight connections between cells, each of which experiences mechanochemical feedback with front-rear polarity, effect well-ordered long-range ERK activation waves.

\section{Discussion}

369 During collective cell migration of epithelial cells, not only leader cells but also follower cells exert traction forces on their substrates to drive cell movement, resulting in long-distance coordinated cell migration (Trepat et al., 2009). Thus, the follower cells can sense the direction of the leader cell migration. Previous studies have revealed that the directional cues are transmitted to the follower cells by intercellular mechanical forces (Serra-Picamal et al., 2012). The mechanical tension varies dynamically and spreads over long distances, leading to establishment of local anisotropic stress, along which the cells tend to migrate (Das et al., 2015; Tambe et al., 2011). However, the molecular mechanism by which the mechanical forces are sustainably transmitted over long distances to direct collective cell migration remains to be revealed. Here we identified that ERK is a key molecule regulating cellular mechanochemical feedback, which governs long-distance sustained propagation of the directional cues. We

380 propose a mechanism of ERK and mechanical force-mediated intercellular communication underlying the collective cell migration (Figure S4G). In the initial process of collective cell 
382 migration, advancement of the leader cells toward the free space exerts pulling forces on the 383 adjacent follower cells, directing front-rear cell polarization. The pulling force then stretches 384 the follower cells and activates the EGFR-ERK signaling cascade, which in turn generates contractile forces at the rear side of the follower cells through the ROCK pathway. The contraction of the follower cells exerts pulling forces on the next follower cells, directing frontrear polarization in the next follower cells and likely providing space for cryptic lamellipodia to protrude for active migration (Das et al., 2015; Farooqui and Fenteany, 2005). At the same time, the pulling force evokes another round of cell stretch, ERK activation, and following ERK-mediated rear contraction in the next follower cells. Thus, intercellular coupling of the ERK-mediated mechanochemical feedback via cell-cell junctions enables sustained propagation of pulling forces, front-rear cell polarity, and ERK activation over a large tissue scale, leading to collective cell migration.

The combination of optogenetic ERK activation and traction force measurement revealed that ERK plays a critical role in translocating cellular force generators. Our experiments demonstrate that the ERK activation triggers cell contraction through ROCK activation (Figure 3B-3E) while it decreases the traction forces (Figures 3L and 3M). The effect of ERK activation on the force generation can be explained by the translocation of dense F-actin fibers from cell bases to cell-cell junctions (Figures $3 \mathrm{O}$ and 3P), which should lead to an efficient tug on adjacent cells. It is plausible that the ERK-induced cell contraction through ROCK involves Rho GEF activation localized at cell-cell junctions. Along this line of reasoning, a previous study demonstrated that mechanical stretching of cells caused localization of Rho GEF at cellcell junctions, giving rise to local activation of RhoA, and thereby the junction-associated actomyosin (Acharya et al., 2018). On the other hand, the decrease in traction forces by ERK activation could be attributed to disruption of focal adhesion-associated actin stress fibers due to promotion of focal adhesion turnover (Colo et al., 2012; Webb et al., 2004). The focal adhesion turnover should increase deformability of cells to efficiently contract upon contractile force generation.

Remarkably, our results imply that ERK activation triggers polarized rear contraction in each cell. As the intracellular ERK activation may not be significantly polarized due to rapid ERK diffusion within the cytoplasm (Fujioka et al., 2006), the effect of ERK activation on the

412 polarized rear contraction is explained by an antagonistic activation of Rac 1 and RhoA (Guilluy 413 et al., 2011). Force loading on a cell-cell junction activates Rac1 via dissociation of merlin from 414 the junction, which directs the cell front (Das et al., 2015). The preoccupied Rac1 activation at 
415 the cell front should restrict the activation of RhoA-regulated contractile machineries to the rear 416 side of the cells, leading to polarized rear contraction upon ERK activation. Therefore, ERK 417 should convey the signal to stimulate the contractile machineries within a cell at the back in 418 response to pulling forces at the front.

An important unsolved question is how the mechanical stimuli are converted into signal transduction to activate ERK. We have previously demonstrated that ADAM17 plays a role for the propagation of the ERK activation in MDCK cells (Aoki et al., 2017). It has been proposed that ADAM17 catalyzes the ectodomain shedding of EGFR ligands (Sahin et al., 2004), and the released ligands can bind and activate EGFR in an autocrine or paracrine manner. In this study, we found that stretch of MDCK cells activates EGFR-ERK pathway (Figures 2D and 2E), as reported in other cell types (Kippenberger et al., 2005; Zhang et al., 2007), and the stretch-induced ERK activation requires ADAM proteinase activity (Figure 2C). Therefore, we suppose that the stretch-induced EGFR activation involves the ADAM17-mediated shedding of EGFR ligands. It has been shown that ERK activates ADAM 17 by means of phosphorylation of Thr735 (Diaz-Rodriguez et al., 2002; Li et al., 2018). However, EGFR activation occurs as early as $1 \mathrm{~min}$ after stretching and precedes ERK activation (Figures 2D-2H), which is at odds with the involvement of ERK activation in the stretch-induced EGFR activation. It has also been reported that src, a protein tyrosine kinase, is involved in ADAM17 activation (Maretzky et al., 2011b; Niu et al., 2015) and is required for the stretch-induced EGFR activation (Zhang et al., 2007). However, we do not know whether ADAM17 is activated upon stretch to cause the ligand shedding. Another possible mechanism is that EGFR ligands are constitutively shed by ADAM proteinases and the sensitivity of EGFR to the ligands may be increased upon stretch. Thus, future studies will be necessary to explore how the mechanical stimuli activate EGFR in a manner dependent on the ligands in the ERK activation waves. Also, we have recently obtained preliminary data showing that ADAM17 is involved in ERK-induced cell contraction as well as stretch-induced ERK activation. Therefore, further investigations should focus on the regulation of ERK activation waves by ADAM17 through multi-tiered system.

In conclusion, we have revealed that mechanical force transmission functions as a mediator of the intercellular ERK signal transduction underlying collective cell migration. 444 Current understanding of the intercellular signal transduction mostly emphasizes the importance of intercellular transfer of biochemical molecules including growth factors, hormones, and neurotransmitters. Thus, our present study raises another consideration, which is the critical role of cellular response to mechanical stimuli in intercellular signal transduction. 


\section{Acknowledgements}

451 This work was supported by JSPS KAKENHI Grant Numbers 17J02107, 15H05949

452 "Resonance Bio", by the SPIRITS 2018 of Kyoto University, and by the Kyoto University Live 453 Imaging Center. We thank Manuel Gómez González regarding the analysis of traction force

454 microscopy, Hiroko Uchida for illustration in Figure S4 and the graphical abstract, James-Alan 455 Hejna for English editing, Daniel Boocock, Edouard Hannezo, and Naoki Honda for fruitful 456 discussions.

457

458

\section{Author contributions}

460 Conceptualization, N.H., M.M., T.H.; Methodology, N.H., K.A., X.T., M.M., T.H.; Software, 461 N.H., X.T., T.H.; Validation, N.H., L.R., A.M.L., T.H.; Formal analysis, N.H., T.H.; 462 Investigation, N.H., L.R., A.M.L.; Resources, N.H., K.A., X.T., T.H.; Data curation, N.H., T.H.; 463 Writing - original draft, N.H., M.M., T.H.; Writing - review \& editing, N.H., K.A., M.M., T.H.; 464 Visualization, N.H., T.H.; Supervision, M.M., T.H.; Project administration, M.M., T.H.; 465 Funding acquisition, N.H., X.T., M.M., T.H.

\section{Declaration of interests}

469 The authors declare no competing interests. 
471

472

473

474

475

476

477

478

479

480

481

482

483

484

485

486

487

488

489

490

491

492

493

494

495

496

497

498

499

500

501

502

503

\section{Main figure titles and legends}

Figure 1. Cell deformation waves precede ERK activation waves during collective cell migration

(A) Phase contrast images (upper), FRET/CFP ratio indicating ERK activity (middle), and strain rate in the direction of collective cell migration (x-strain rate; lower) are represented at $1 \mathrm{~h}$ (left), $8 \mathrm{~h}$ (center), and $14 \mathrm{~h}$ (right) after start of time-lapse imaging. In the x-strain rate images, extending and shrinking regions are shown in red and blue, respectively. Scale bar, 200 $\mu \mathrm{m}$. The imaging interval is $1 \mathrm{~min}$.

(B and C) x-t kymographs of ERK activity (B) and x-strain rate (C), from (A). White arrows indicate the rightward propagation of ERK activation waves (B) and cell deformation waves (C).

(D) Temporal change of ERK activation rate and $\mathrm{x}$-strain rate in a representative cell.

(E) Temporal cross-correlations between x-strain rate and ERK activation rate. The blue line indicates the average temporal cross-correlation coefficients with standard deviations (SDs). $n$ $=212$ cells from three independent experiments. See also Movie S1.

\section{Figure 2. Passive cell extension triggers ERK activation via EGFR signaling}

(A and B) ERK activity change in MDCK cells on an elastic chamber. (A) Representative images before (left) and $20 \mathrm{~min}$ after uniaxial 50\% stretch (right). The black double-headed arrow indicates the axis of the uniaxial stretch. White and yellow arrowheads each correspond to the same cells in the left and right images. Scale bar, $30 \mu \mathrm{m}$. (B) Violin plots of ERK activity 20 min after $20-50 \%$ stretch or that without stretch ( $0 \%$ stretch). Red and gray lines indicate median and mean values, respectively. $n=3612$ cells ( $0 \%$ stretch), 4771 cells ( $20 \%$ stretch), 3856 cells ( $30 \%$ stretch), 4070 cells ( $40 \%$ stretch), and 3178 cells (50\% stretch) from three independent experiments. Dunnett's test; $P=0.0969$ ( 0 versus $20 \%$ stretch), $* * * * P<0.0001$ (0 versus 30,40 , or $50 \%$ stretch).

(C) ERK activity changes in cells treated with DMSO, $1 \mu \mathrm{M}$ PD153035, and $10 \mu \mathrm{M}$ marimastat, from immediately after to $20 \mathrm{~min}$ after the stretch are shown as means with SDs. $n=3634$ cells (DMSO), 3237 cells (PD153035), and 4000 cells (marimastat) from three independent experiments. Dunnett's test, $* * * * P<0.0001$.

(D) MDCK cells were stretched and then lysed at the indicated time points. The cell lysates were analyzed by immunoblotting with the indicated antibodies. 
$504(\mathrm{H})$ are represented as means with SDs $(n=3)$.

505 See also Figure S1.

506

Figure 3. ERK activation triggers cell contraction via Rho-associated kinase activation

508 (A) Schematics of an experiment with the light-inducible system. The boundary between the cell populations with and without 2paRAF expression was imaged.

(B) Representative images of CFP (EKAREV-NLS; upper) and CIBN-mScarlet-I-CAAX

511 (2paRAF; middle) treated with DMSO (left), $200 \mathrm{nM}$ trametinib (center), and $10 \mu \mathrm{M}$ Y-27632

512 (right) were obtained at the boundary between the cells with and without 2paRAF. Lower panels

513 indicate kymographs of the CIBN-mScarlet-I-CAAX (2paRAF) images. Blue lines indicate the

514 blue light illumination. Scale bar, $30 \mu \mathrm{m}$. The imaging interval is $5 \mathrm{~min}$.

515 (C) Displacement of the boundary is plotted over time after blue light exposure. The lines 516 represent the average with SDs. $n=9$ from three independent experiments.

517 (D) ROCK activity images of MDCK cells expressing 2paRAF and a ROCK biosensor are 518 represented at $0 \mathrm{~min}$ (left) and $15 \mathrm{~min}$ (right) after the start of blue light exposure. Scale bar, 20 $519 \mu \mathrm{m}$. The imaging interval is $1 \mathrm{~min}$.

520 (E) Quantification of ROCK activity in each cell in (D) after the start of blue light exposure. 521 The line represents the average with SDs. $n=15$ from three independent experiments. Unpaired $522 t$-test, $P=0.0065$.

523 (F) ROCK activity images are represented at $0 \mathrm{~h}$ (upper) and $12 \mathrm{~h}$ (lower) after removal of 524 confinement. Scale bar, $200 \mu \mathrm{m}$. The imaging interval is $2 \mathrm{~min}$.

525 (G) A kymograph of the ROCK activity in (F). White arrows indicate the rightward propagation 526 of ROCK activity.

527 (H and J) A kymograph of ROCK activity is shown before and after $200 \mathrm{nM}$ trametinib $(\mathrm{H})$ and $528100 \mu \mathrm{M}$ Y-27632 $(\mathrm{J})$ treatment. The imaging interval is $2 \mathrm{~min}$.

529 (I and K) ROCK activity in 5 representative cells was plotted over time after trametinib (I) and $530 \quad \mathrm{Y}-27632(\mathrm{~K})$ treatment.

531 (L) Traction force microscopy of the cell seeded on polyacrylamide gels with optogenetic ERK 532 activation. Differential interference contrast (DIC) images (upper), CIBN-mScarlet-I-CAAX 533 fluorescence (2paRAF; middle), and traction force (lower) are represented at $-1 \mathrm{~h}$ (left), $1 \mathrm{~h}$ 534 (center), and $4 \mathrm{~h}$ after the start of blue light exposure. Scale bar, $50 \mu \mathrm{m}$. The imaging interval 535 is $5 \mathrm{~min}$.

536 (M) Mean traction force in cells with and without 2paRAF, as shown in (L), with SDs (n=3). 
537 (N) Mean traction force in cells with 2paRAF with DMSO or $200 \mathrm{nM}$ trametinib treatment at 2 $538 \mathrm{~h}$ after the start of blue light exposure $(\mathrm{n}=3)$.

539 (O) Fluorescence images of F-actin (phalloidin) in migrating MDCK cells on collagen-coated 540 glass substrates $45 \mathrm{~min}$ after treatment with DMSO, $100 \mathrm{ng} \mathrm{mL}{ }^{-1} \mathrm{EGF}, 200 \mathrm{nM}$ trametinib, or 541 both EGF and trametinib. Scale bar, $10 \mu \mathrm{m}$.

542 (P) Intensity profile of F-actin along the orange lines in (O). Orange arrowheads indicate edges 543 of cells.

544 See also Figure S2, Movie S3 and S4.

545

\section{Figure 4. ERK activation waves are mediated by intercellular mechanical force}

547 (A) Schematics of an experiment using the rapamycin-inducible system. The boundary between 548 the cell population with RA Rho GEF and that with EKAREV-NLS expression was imaged.

549 (B) DIC images (upper), ERK activity (middle), and ERK wave directionality (lower) are represented at $0 \mathrm{~h}$ after DMSO (left) and $50 \mathrm{nM}$ rapamycin (right) treatment. Broken lines indicate the cell population boundary. Arrows represent the direction of ERK activity propagation. For the analysis of ERK wave directionality, the EKAREV-NLS-expressing cells located to the left of the cell population boundary were omitted from the analysis. Scale bar, $554 \quad 100 \mu \mathrm{m}$. The imaging interval is $5 \mathrm{~min}$.

555 (C) ERK activity (upper) and ERK wave directionality (lower) are represented at $13 \mathrm{~h}$ after the 556 drug treatment.

557 (D) Kymographs of ERK activity with DMSO (left) or rapamycin (right) treatment. The 558 EKAREV-NLS-expressing cells located on the left side of the cell population boundary were 559 excluded from the kymographs for visibility.

560 (E and F) Polar histograms showing the distribution of ERK wave (E) and cell displacement 561 (F) direction over 13-13.5 h after treatment with DMSO (left) and rapamycin (right). For ERK 562 wave direction, $n=1078$ (DMSO) and 1127 (rapamycin) from three independent experiments. 563 For cell displacement direction, $n=475$ (DMSO) and 1167 (rapamycin) from three independent 564 experiments.

565 (G) Analysis of the expression level of $\alpha$-catenin and $\beta$-actin in WT and $\alpha$-catenin KO MDCK 566 cells by immunoblotting.

567 (H) DIC images (upper) and immunofluorescence images of E-cadherin (lower) in the confluent 568 WT and $\alpha$-catenin KO MDCK cells.

569 (I) The ERK activity 16 min after the addition of EGF was plotted as $\log ($ dose) response. Orange 
570 circles (WT cells) and blue squares ( $\alpha$-catenin KO cells) represent the average of ERK activity

571 with SDs. The data were obtained from three independent experiments.

$572(\mathrm{~J}$ and $\mathrm{K})$ Kymographs of ERK activity during collective cell migration in WT $(\mathrm{J})$ and $\alpha$-catenin $573 \mathrm{KO}(\mathrm{K}) \mathrm{MDCK}$ cells. The imaging interval is $5 \mathrm{~min}$.

$574 \quad$ See also Movie S5 and S6.

575

Figure 5. Multicellular alignment of front-rear polarization underpins unidirectional

\section{ERK activation waves}

578 (A) A representative cell expressing mCherry in the cytosol is marked by a broken line. The left 579 column shows composite images of phase contrast and mCherry fluorescence (green), and the 580 right column shows ERK activity. Cells showed low ERK activity in the contracted state (upper 581 and lower) and high ERK activity in the extended state (middle).

582 (B) Changes in lengths of cells along the $\mathrm{x}$ axis and $\mathrm{y}$ axis from contracted (minimum ERK activity) to extended (maximum ERK activity) states are plotted. Each line indicates an individual cell. $n=30$ cells from three independent experiments. Paired $t$-test, $* * * * P<0.0001$. (C) Changes in lengths of cells along the $\mathrm{x}$ axis and $\mathrm{y}$ axis from extended (maximum ERK activity) to contracted (minimum ERK activity) states are plotted. Each line indicates an individual cell. $n=30$ cells from three independent experiments. Paired $t$-test, $* * * * P<0.0001$. (D) Immunofluorescence images of F-actin (left) and di-phosphorylated MLC (ppMLC; center) at the basal plane in MDCK cells at $17 \mathrm{~h}$ after migration. The right column indicates the composite images of F-actin and ppMLC.

591 (E) Immunofluorescence images of the Golgi apparatus (GM130) and the nucleus (EKAREV592 NLS) in MDCK cells at $21 \mathrm{~h}$ after migration. The upper image shows a wide-view field, and 593 the lower images are magnified images of the regions corresponding to the numbered windows 594 in the upper image.

595 (F) Polar histograms showing the distribution of Golgi orientation relative to the nucleus in the cells at 0-0.5 $\mathrm{mm}$ (left), 0.5-1.0 (center), and greater than $1.0 \mathrm{~mm}$ (right) distant from the leader cells. $n=1297$ cells $(0-0.5 \mathrm{~mm}), 2096$ cells $(0.5-1.0 \mathrm{~mm})$, and 1452 cells $(>1.0 \mathrm{~mm})$ from three 598 independent experiments.

599 (G) $\theta$ is the angle between the reference direction and sample direction. Directedness was 600 calculated by the indicated equation. The reference direction is set to the left for analysis of 601 Golgi orientation and cell migration direction, and to the right for ERK wave direction, in order to match the signs of their directedness. 
603 (H-J) Mean directedness of Golgi orientation (H), ERK wave direction (I), and cell migration 604 (J) binned every $300 \mu \mathrm{m}$ from the leader cells after $0 \mathrm{~h}, 6 \mathrm{~h}$, and $21 \mathrm{~h}$ migration is plotted over 605 the distance from the leader cells, with SDs. The data were obtained from three independent 606 experiments. The first bin of the ERK wave directedness was excluded from the result because 607 of outliers due to boundary effects.

608 (K) Immunofluorescence images of Golgi (GM130) and nuclei (EKAREV-NLS) in shRac1609 and shCdc42-expressing MDCK cells at regions greater than $1 \mathrm{~mm}$ from the leader cells after $61021 \mathrm{~h}$ migration.

611 (L-N) Directedness of Golgi orientation (Dunnett's test, $* * * P=0.0001(0.6-0.9 \mu \mathrm{m}$, WT versus 612 shCdc42), $P=0.0508(0.9-1.2 \mathrm{~mm}$, WT versus $\operatorname{shCdc} 42), * * * P=0.0009(0.6-0.9 \mu \mathrm{m}, \mathrm{WT}$ 613 versus shRac1), $P=0.2023(0.9-1.2 \mu \mathrm{m}$, WT versus shRac1)) (L), ERK wave direction 614 (Dunnett's test, $* * P=0.0067$ (0.9-1.2 mm, WT versus shCdc42), $* P=0.0290(1.2-1.5 \mathrm{~mm}$, 615 WT versus shCdc42), ${ }^{* *} P=0.0019(0.9-1.2 \mathrm{~mm}$, WT versus $\operatorname{shRac} 1),{ }^{*} P=0.0248(1.2-1.5$ $616 \mathrm{~mm}$, WT versus shRac1)) (M), and cell migration direction (Dunnett's test, ${ }^{*} P=0.0238$ (1.2$6171.5 \mathrm{~mm}$, WT versus shRac1), $P=0.0655(1.2-1.5 \mathrm{~mm}$, WT versus shCdc42)) $(\mathrm{N})$ binned every $618300 \mu \mathrm{m}$ from the leader cells after $21 \mathrm{~h}$ migration were plotted over the distance from the leader 619 cells with SDs. The data of WT cells is the same as that of $21 \mathrm{~h}$ migration in (H-J). The data 620 were obtained from three independent experiments.

621 See also Figure S3 and Movie S7.

622

Figure 6. Synthetic ERK activation waves orient front-rear polarity in follower cells

624 (A) Immunofluorescence images of Golgi (GM130) and nuclei (EKAREV-NLS) in MDCK 625 cells treated with DMSO (left), $200 \mathrm{nM}$ trametinib (center), and $10 \mathrm{nM}$ TPA (right) at a region 626 more than $1.0 \mathrm{~mm}$ distant from the leader cells at $21 \mathrm{~h}$ after migration. Scale bar, $100 \mu \mathrm{m}$.

627 (B and C) Mean directedness of Golgi orientation (B) and cell migration direction (C) binned 628 every $300 \mu \mathrm{m}$ from the leader cells after $21 \mathrm{~h}$ migration were plotted over distance from the leader cells, with SDs. The data were obtained from three independent experiments.

630 (D) Flow diagram of an experiment on synthetic ERK activation waves.

631 (E) Fluorescence images of Golgi apparatus (Golgi-7-mCherry) and nuclei (EKAREV-NLS) in 632 MDCK cells with or without optogenetic ERK activation waves for $18 \mathrm{~h}$. The upper images are 633 wide-view fields, and the lower images are magnified views of the regions outlined by white 634 windows in the upper images.

635 (F) The mean directedness of Golgi orientation binned every $100 \mu \mathrm{m}$ from the leader cells in 
636 (E) was plotted over distance from the leader cells, with SDs. The data were obtained from 637 three independent experiments. Unpaired $t$-test; $* * P=0.0011(0.6-0.7 \mu \mathrm{m}$, plus versus minus), $638 * P=0.0267(0.7-0.8 \mathrm{~mm}$, plus versus minus).

639 See also Figure S4, Movie S8 and S9.

640

641 Figure 7. Modeling cellular mechanochemical feedback with polarity demonstrates long642 range unidirectional ERK activation waves

643 (A) Schematics of the mathematical model.

644 (B) Contraction mode depending on the parameter $\omega$, defined as the polarity level.

645 (C) Snapshot of ERK activity in the CPM simulation. Scale bar, $200 \mu \mathrm{m}$. (D) Kymograph of 646 ERK activity along the $\mathrm{x}$-axis and simulation step. The color corresponds to values of the color 647 bar in (C).

648 (E) Spatial distribution of mean directedness, with SDs. The color shows different simulation 649 steps. $n=5$.

650 (F) The effect of polarity level $\omega$ on the directedness. The color represents mean values of the 651 directedness. $n=5$.

652 (G and $\mathrm{H})$ Cell polarity directedness with $(+)$ or without $(-)$ ERK wave. The color in $(\mathrm{G})$ 653 represents the angle of cell polarity. $(\mathrm{H})$ Mean polarity directedness from the leading edge, with 654 SDs. $n=5$.

655 (I) Snapshot of ERK activity distribution in weak $(\gamma=0.5)$ and strong $(\gamma=1.5)$ multicellular 656 integrity.

657 (J) Mean y-axis spatial correlation length of the ERK activity on the intercellular integrity $\gamma$, 658 with SDs. $n=5$. See also Movie S10.

659 
660

661

662

663

664

665

666

667

668

669

670

671

672

673

674

675

676

677

678

679

680

681

682

683

684

685

686

687

688

689

690

691

692

STAR Methods

\section{RESOURCE AVAILABILITY}

\section{Lead Contact}

Further information and requests for resources and reagents should be directed to and will be fulfilled by the Lead Contact, Tsuyoshi Hirashima (hirashima.tsuyoshi.2m@kyoto-u.ac.jp).

\section{Materials Availability}

Materials developed for this study are available on request to the corresponding authors.

\section{Data and Code Availability}

Data collected and computer codes developed for this study are available on request to the corresponding authors.

\section{EXPERIMENTAL MODEL AND SUBJECT DETAILS}

\section{Cell culture}

MDCK cells were from the RIKEN BioResource Center (no. RCB0995). Lenti-X 293T cells were purchased from Clontech (no. 632180, Mountain View, CA). These cells were maintained in D-MEM (no. 044-29765, Wako, Osaka, Japan) supplemented with 10\% FBS (no. 172012500ML, SIGMA, St. Louis, MO), 100 unit $\mathrm{mL}^{-1}$ penicillin, and $100 \mu \mathrm{g} \mathrm{mL}^{-1}$ streptomycin (no. 26253-84, Nacalai Tesque, Kyoto, Japan) in a 5\% $\mathrm{CO}_{2}$ humidified incubator at $37^{\circ} \mathrm{C}$.

\section{Establishment of stable cell lines}

For the establishment of EKAREV-NLS-expressing MDCK cells, a Tol2 transposon system was used. MDCK cells were co-transfected with pT2A-EKAREV-NLS and pCAGGS-T2TP encoding Tol2 transposase, and sorted by FACS as previously described (Kawabata and Matsuda, 2016; Sakurai et al., 2012). To establish MDCK cells stably expressing 2paRAF, pT2ADWpuro_2paRAF or pT2ADWpuro_2paRAFAmScarlet-I were used for the Tol2 transposon system. For the generation of cells stably expressing Eevee-ROCK-NES and the other ectopic proteins, a lentiviral or retroviral system was employed. To prepare the lentivirus, pCSII-based lentiviral vector (Miyoshi et al., 1998) or lentiCRISPRv2 (Addgene Plasmid: no. 52961), psPAX2 (Addgene Plasmid: no. 12260), and pCMV-VSV-G-RSV-Rev were cotransfected into Lenti-X 293 T cells using polyethylenimine (no. 24765-1, Polyscience Inc., 
693 Warrington, PA). To prepare the retrovirus, pCX4-based retroviral vector (Akagi et al., 2003)

694 or pSUPER (Oligoengine, Seattle, WA), pGP, and pCMV-VSV-G-RSV-Rev were co695 transfected into Lenti-X 293T cells. Stable cell lines of MDCK cells were selected and 696 maintained in media containing the following antibiotics: MDCK/EKAREV-NLS/2paRAF, 4 $697 \mu \mathrm{g} \mathrm{mL}^{-1}$ puromycin (no. P-8833, SIGMA); MDCK/EKAREV-NLS/2paRAF/Golgi-7-mCherry, $6984 \mu \mathrm{g} \mathrm{mL}^{-1}$ puromycin and $10 \mu \mathrm{g} \mathrm{mL}^{-1}$ blasticidin S (no. 029-18701, Wako); MDCK/Eevee699 ROCK-NES, $10 \mu \mathrm{g} \mathrm{mL}{ }^{-1}$ blasticidin S; MDCK/Eevee-ROCK-NES/2paRAF, $10 \mu \mathrm{g} \mathrm{mL}{ }^{-1}$ 700 blasticidin $\mathrm{S}$ and $4 \mu \mathrm{g} \mathrm{mL} \mathrm{mL}^{-1}$ puromycin; MDCK/EKAREV-NLS/mCherry, $4 \mu \mathrm{g} \mathrm{mL} \mathrm{m}^{-1}$ 701 puromycin; MDCK/RA Rho GEF, $4 \mu \mathrm{g} \mathrm{mL}{ }^{-1}$ puromycin and $10 \mu \mathrm{g} \mathrm{mL}^{-1}$ blasticidin S.

702

703

\section{CRISPR/Cas9-mediated KO cell lines}

704 For CRISPR/Cas9-mediated KO of dog CTNNA1 ( $\alpha$-catenin), single guide RNAs (sgRNA) 705 targeting the exon were designed using the CRISPRdirect (Naito et al., 2015). The following sequence was used for the sgRNA sequence: GTAGAAGATGTTCGAAAACA. Oligo DNAs for the sgRNA were cloned into the lentiCRISPRv2 vector, and the sgRNA and Cas9 were introduced into MDCK cells by lentiviral infection. The infected cells were selected with 4.0 $\mu \mathrm{g} \mathrm{mL}^{-1}$ puromycin. After the selection, reduction in expression levels of the proteins was confirmed by immunoblotting. Bulk cells were used for the experiments.

\section{shRNA-mediated KD cell line}

713 For shRNA-mediated KD of $\operatorname{dog} R A C 1$ and $C D C 42$, the DNA oligomers corresponding to the 714 shRNAs targeting the genes were subcloned into pSUPER vector. The following sequences were used for shRNA target sequences: RAC1, GCCTTCGCACTCAATGCCAAG; CDC42, GAACAAACAGAAGCCTATC. The shRNAs were introduced into MDCK cells by retroviral infection. The infected cells were selected with $4.0 \mu \mathrm{g} \mathrm{mL}^{-1}$ puromycin. After the selection, reduction in expression levels of the target proteins was confirmed by immunoblotting.

\section{METHOD DETAILS}

\section{$721 \quad$ Plasmids}

722 pT2A-EKAREV-NLS and plasmids for RA Rho GEF (pCX4puro-LDR, and pCX4bsr-3HAFKBP-p63RhoGEF-DH) were described previously (Kawabata and Matsuda, 2016; Komatsu et al., 2011; Li et al., 2017; Urasaki et al., 2006; van Unen et al., 2015). pT2ADWpuro_2paRAF 
726 described previously (Kinjo et al., 2019). pT2ADWpuro_2paRAF $\Delta m$ Scarlet-I encoding 727 2paCRY2-RAF1-P2A-CIBN-CAAX was generated by PCR and subcloned into the 728 pT2ADWpuro vector. The FRET biosensor for ROCK activity, Eevee-ROCK-NES, was 729 described previously (Li et al., 2017) and subcloned into a pCSII vector. pCSII and pCMV730 VSVG-RSV-Rev were kindly gifted from H. Miyoshi (RIKEN BioResource Center, Ibaraki, 731 Japan). pGP was gifted from T.Akagi. psPAX2 was the kind gift of Didier Trono (Addgene plasmid: no. 12260). mCherry-Golgi-7 was a gift from Michael Davidson (Addgene plasmid: no. 55052) and the cassette was subcloned into a pCSIIbsr vector. pCAGGS-T2TP was a gift from Koichi Kawakami (National Institute of Genetics, Shizuoka, Japan).The cDNA of mCherry was subcloned into a pCSIIpuro vector to generate pCSIIpuro-mCherry.

\section{Reagents and antibodies}

738 The following reagents were used: trametinib (no. T-8123, LC Laboratories, Woburn, MA), Y27632 (no. 253-00513, Wako, Osaka, Japan), PD153035, marimastat (no. SC-202223, Santa Cruz Biotechnology, Dallas, TX), rapamycin (no. R-5000, LC Laboratories), TPA (no. P-1680, LC Laboratories), Rhodamine Phalloidin (no. R415, Invitrogen, Carlsbad, CA, 1:40 dilution for immunofluorescence), EGF (no. E9644, SIGMA, St. Louis, MO).

The following primary and secondary antibodies were used for immunoblotting: AntiEGFR rabbit antibody (no. 4267, Cell Signaling Technology, Danvers, MA, 1:1,000 dilution); anti-phospho-EGFR (Tyr1068) rabbit antibody (no. 3777, Cell Signaling Technology, 1:1,000 dilution); anti-RAF1 mouse antibody (no. 610152, BD Biosciences, Franklin Lakes, NJ, 1:1,000 dilution); anti-phospho-RAF1 (Ser338) rabbit antibody (no. 9427, Cell Signaling Technology, 1:1,000 dilution); anti-MEK1/2 rabbit antibody (no. 9122, Cell Signaling Technology, 1:1,000 dilution); anti-phospho-MEK1/2 (Ser217/221) rabbit antibody (no. 9121,

750 Cell Signaling Technology, 1:1,000 dilution); anti-ERK1/2 mouse antibody (no. 610123, BD 751 Biosciences, 1:2,000 dilution); anti-phospho-p44/42 MAPK (Erk1/2; Thr202/Tyr204) rabbit

752

753 antibody (no. 9101, Cell Signaling Technology, 1:2,000 dilution); anti- $\beta$-Actin rabbit antibody (no. 4970, Cell Signaling Technology, 1:1,000 dilution); anti-Cdc42 mouse antibody (no. 610929, BD Biosciences, 1:1,000 dilution); anti-Rac1 mouse antibody (no. 610650, BD Biosciences, 1:1,000 dilution); anti- $\alpha$-catenin mouse antibody (no. 610194, BD Biosciences, 1:1,000 dilution); IRDye 680-conjugated goat anti-mouse IgG antibody (no. 926-32220, LICOR Biosciences, Lincoln, NE, 1:10,000 dilution); and IRDye 800CW goat anti-rabbit IgG antibody (no. 926-32211, LI-COR Biosciences, 1:10,000 dilution). 
The following primary and secondary antibodies were used for immunofluorescence: anti-phospho-Myosin Light Chain 2 (Thr18/Ser19) rabbit antibody (no. 3674, Cell Signaling Technology, 1:50 dilution); anti-E-cadherin rabbit antibody (no. 3195, Cell Signaling Technology, 1:300 dilution); Alexa 647-conjugated goat anti-mouse $\operatorname{IgG}(\mathrm{H}+\mathrm{L})$ antibody (no. A-21235, Thermo Fisher Scientific, Waltham, MA, 1:1,000 dilution); alexa 647-conjugated goat anti-rabbit IgG $(\mathrm{H}+\mathrm{L})$ antibody (no. A-21245, Thermo Fisher Scientific, 1:1,000 dilution); and alexa 568-conjugated goat anti-rabbit $\operatorname{IgG}(\mathrm{H}+\mathrm{L})$ antibody (no. A-11036, Thermo Fisher Scientific, 1:1,000 dilution).

\section{Confinement release assay}

To confine the MDCK cell monolayer, a Culture-Insert 2 Well (no. 81176, ibidi, Martinsried, Germany) was placed on a glass-bottom dish coated with $0.3 \mathrm{mg} \mathrm{mL}^{-1}$ type I collagen (Nitta Gelatin, Osaka, Japan). MDCK cells $\left(7 \times 10^{3}\right.$ cell $)$ were seeded in the Culture-Insert. $24 \mathrm{~h}$ after seeding, the Culture-Insert was removed, and the medium was replaced with Medium 199 (11043023; Life Technologies, Carlsbad, CA) supplemented with 10\% FBS, 100 unit $\mathrm{mL}^{-1}$ penicillin, and $100 \mu \mathrm{g} \mathrm{mL}^{-1}$ streptomycin. $30 \mathrm{~min}$ after the removal of the Culture-Insert, the cells were imaged with an epifluorescence microscope every 1 to $10 \mathrm{~min}$.

\section{Boundary assay}

For the light-induced ERK activation experiment, MDCK/EKAREV-NLS/2paRAF cells were seeded in a well of a Culture-Insert 2 well placed on a 24 well glass bottom plate, and MDCK/EKAREV-NLS cells were seeded in the other well of the insert and outside of the insert. After $15 \mathrm{~h}$ incubation, the insert was removed, followed by further incubation for $36 \mathrm{~h}$, allowing the cells to fill the gap between the cell populations. The interface between cells with and without 2paRAF expression was imaged, and the cells were exposed to $438 \mathrm{~nm}$ blue LED light every 5 min for CRY2 activation.

For the drug-induced cell contraction experiment, a well of the insert was removed by cutting, and the remained insert with a well was placed on a 24 well glass-bottom plate. MDCK cells expressing RA Rho GEF were seeded in the well. After $6 \mathrm{~h}$ incubation, the insert was removed, and MDCK/EKAREV-NLS cells were plated in the 24 well glass-bottom plate. The interface between cells with and without RA Rho GEF expression was imaged, and dimethyl sulfoxide (DMSO; final $0.1 \%$ ) or rapamycin (final $50 \mathrm{nM}$ ) was added into the medium for the Rho GEF activation. To determine the ERK wave directionality, heat maps of ERK activity 
792 were obtained by interpolating the signals in regions between the nuclei of MDCK/EKAREV793 NLS cells in the FRET/CFP ratio images. The heat maps of ERK activity were analyzed by 794 particle image velocimetry (PIV) using a free Matlab-toolbox, MatPIV (Sveen, 2004), to 795 calculate the ERK wave directionality. The size and overlap of the interrogation window was 796 set to $349 \mu \mathrm{m}$ and $75 \%$, respectively. To determine the directionality of cell displacement, the 797 Fiji TrackMate plugin (Jaqaman et al., 2008; Schindelin et al., 2012) was applied to the CFP 798 fluorescence images for tracking each cell over 13-13.5 h after treatment with DMSO or 799 rapamycin.

800

\section{Cell stretch assay}

802 For the cell stretch assay, MDCK cells $\left(2 \times 10^{5}\right.$ cell $)$ were seeded on an elastic silicone chamber 803 (no. STB-CH-04, STREX, Osaka, Japan) coated with $0.3 \mathrm{mg} \mathrm{mL}^{-1}$ type I collagen. After $24 \mathrm{~h}$ 804 incubation, the MDCK cells on the stretch chamber were uniaxially stretched by $20-50 \%$ with 805 a manual cell-stretching system (no. STB-100-04, STREX) on an epifluorescence microscope. 806 The ERK activity 20 min after $20-50 \%$ stretch or that without stretch ( $0 \%$ stretch) was shown 807 as violin plots by using a MATLAB code, Violin Plot (Hoffmann, 2020). Red and gray lines 808 indicate median and mean values. For immunoblotting, stretched MDCK cells were lysed with 809 SDS sample buffer containing $62.5 \mathrm{mM}$ Tris-HCl (pH6.8), 12\% glycerol, 2\% SDS, $40 \mathrm{ng} \mathrm{mL}^{-1}$ 810 bromophenol blue, and 5\% 2-mercaptoethanol, followed by sonication with a Bioruptor UCD811200 (Cosmo Bio, Tokyo, Japan). After boiling at $95^{\circ} \mathrm{C}$ for $5 \mathrm{~min}$, the samples were resolved by 812 SDS-PAGE on SuperSep Ace 5-20\% precast gels (Wako), and transferred to PVDF membranes 813 (Merck Millipore, Billerica, MA). All antibodies were diluted in Odyssey blocking buffer (LI814 COR Biosciences). Proteins were detected by an Odyssey Infrared Imaging System (LI-COR 815 Biosciences).

\section{Time-lapse imaging}

818 FRET images were obtained and processed under essentially the same conditions and 819 procedures as previously described (Aoki and Matsuda, 2009). Briefly, cells were imaged with 820 an IX83 inverted microscope (Olympus, Tokyo, Japan) equipped with a UPlanFL-PH 10x/0.3 821 (Olympus), a UPlanSApo 20x/0.75 (Olympus), or a UPlanSApo 40x/0.95 objective lens 822 (Olympus), a DOC CAM-HR CCD camera (Molecular Devices, Sunnyvale, CA), a Spectra-X 823 light engine (Lumencor Inc., Beaverton, OR), an IX3-ZDC laser-based autofocusing system 824 (Olympus), an electric XY stage (Sigma Koki, Tokyo, Japan), and a stage top incubator (Tokai 
825

826

827

828

829

830

831

832

833

834

835

836

837

838

839

840

841

842

843

844

845

846

847

848

849

850

851

852

853

854

855

856

857

Hit, Fujinomiya, Japan). The filters and dichromatic mirrors used for time-lapse imaging were as follows: for FRET imaging, a 438/24 excitation filter (incorporated in the Spectra-X light engine), a FF458-Di02-25×36 (Semrock, Rochester, NY) dichromatic mirror, and FF01483/32-25 (Semrock) and FF01-542/27-25 (Semrock) emission filter for CFP and FRET, respectively. For mCherry and mScarlet-I imaging, a 575/25 excitation filter, a glass dichromatic mirror (Olympus), and FF01-624/40-25 (Semrock) emission filters were used.

\section{Immunofluorescence and confocal microscopy}

MDCK cells were fixed with 4\% paraformaldehyde in PBS for $15 \mathrm{~min}$ at room temperature, followed by permeabilization with $0.2 \%$ Triton X-100 in PBS for $5 \mathrm{~min}$. The samples were then incubated with $1 \%$ BSA in PBS for $1 \mathrm{~h}$ at room temperature, followed by sequential incubation with primary and secondary antibodies diluted with $1 \%$ BSA in PBS overnight at $4^{\circ} \mathrm{C}$ (primary antibodies) or for $1 \mathrm{~h}$ at room temperature (secondary antibodies). Images were collected using a Fluoview FV1000 confocal microscope (Olympus) equipped with a UPlanSApo 60x/1.35 or a UPlanSApo 100x/1.40 objective lens (Olympus).

\section{Traction force microscopy}

Polyacrylamide gel substrates were prepared as previously described, with slight modifications (Aoki et al., 2017; Rodriguez-Franco et al., 2017). Briefly, glass-bottom dishes (IWAKI, Shizuoka, Japan) were treated with $2 \%$ acetic acid (WAKO) and $0.2 \%$ 3(Trimethoxysilyl)propyl methacrylate (SIGMA) in 80\% ethanol for $2 \mathrm{~min}$. After the removal of the solution, the dishes were dried for $15 \mathrm{~min}$. For $3 \mathrm{kPa}$ gels, 5.5\% acrylamide, $0.09 \%$ bisacrylamide, $0.05 \%$ ammonium persulfate, $0.05 \% \mathrm{~N}, \mathrm{~N}, \mathrm{~N}^{\prime}, \mathrm{N}^{\prime}$-tetramethyl ethylenediamine, and $0.01 \%$ deep red fluorescent carboxylate-modified beads $(0.2 \mu \mathrm{m}$ diameter; F8810, Thermo Fisher Scientific) in PBS were prepared. $18 \mu \mathrm{L}$ of the solution was put on the glass-bottom dishes and $18 \mathrm{~mm}$ glass cover slips (Matsunami, Osaka, Japan) were placed on top of them. After polymerization, the gels were covered with $2 \mathrm{mg} \mathrm{mL}^{-1}$ Sulfo-SANPAH (no. ab145610, abcam, Cambridge, UK) and activated by ultraviolet light for $5 \mathrm{~min}$ with an Ultraviolet Crosslinker CL-1000 apparatus (UVP, Upland, CA). The procedure was repeated again with 7 min UV irradiation. The gels were coated with $100 \mu \mathrm{m} \mathrm{mL}^{-1}$ type I collagen overnight at $4^{\circ} \mathrm{C}$. Then, the gels were washed three times with PBS and incubated with culture medium for $1 \mathrm{~h}$. For traction force microscopy, the bead fluorescence was imaged with an IX83 inverted microscope equipped with a 632/22 excitation filter (incorporated in the Spectra-X light engine), 
858 a glass dichromatic mirror (Olympus), and FF01-692/40-25 (Semrock) emission filter. A 859 reference image was obtained after the removal of cells by trypsinization. Traction forces were 860 computed by Fourier-transform traction microscopy as described previously (Trepat et al., $8612009)$.

862

863 EGF stimulation assay

864 To evaluate the sensitivity of cells to EGFR stimulation, WT or $\alpha$-catenin KO MDCK cells (2.5 $865 \times 10^{3}$ cell) were seeded on a 96 well glass bottom plate coated with $0.3 \mathrm{mg} \mathrm{mL}^{-1}$ type I collagen. 866 After $24 \mathrm{~h}$ incubation, the MDCK cells were treated with different dose of EGF $(0.01,0.1,1,3$, $\left.86710,100 \mathrm{ng} \mathrm{mL}^{-1}\right)$. The ERK activity $16 \mathrm{~min}$ after the addition of EGF was plotted as $\log (\operatorname{dose})$ 868 response and the plots were fitted to a variable slope model by least squares fit method with 869 GraphPad Prism 7 software.

870

\section{Optogenetic ERK activation wave assay}

872 To illuminate a defined rectangular region with blue light, transillumination light of a 100-W 873 halogen lamp filtered with BA420-460 (Olympus) was covered by a homemade aperture mask 874 containing a slit. The image of the slit was focused on the sample plane by the condenser lens. 875 The width of the focused image of the illumination light was approximately $35 \mu \mathrm{m}$. The 876 illuminated region was moved at $3 \mu \mathrm{m} \mathrm{min}^{-1}$ velocity, and the illumination was patterned with $877400 \mu \mathrm{m}$ spatial intervals at each time point. The procedure was applied to the migrating $878 \mathrm{MDCK} / \mathrm{EKAREV-NLS/2paRAF/Golgi-7-mCherry} \mathrm{cells} \mathrm{treated} \mathrm{with} 1 \mu \mathrm{M}$ PD153035, an 879 EGFR inhibitor, to suppress autonomous ERK activation waves.

880

\section{Kymography}

882 To obtain the kymographs of FRET/CFP ratios and $\mathrm{x}$-strain rate, these values were averaged 883 along the $y$-axis in a defined region of the images, providing an intensity line along the $\mathrm{x}$-axis. 884 The operation was repeated for the respective time points, and the intensity lines were stacked 885 along the $y$-axis for all time points.

886

\section{$887 \quad$ Mathematical modeling}

888 A cellular Potts model for cell migration

889 We used a two-dimensional cellular Potts model (CPM), also known as a Glazier-Graner890 Hogeweg model, widely used for multicellular dynamics (Balter et al., 2007; Krieg et al., 2008; 
891 te Boekhorst et al., 2016; van Helvert et al., 2018). In the CPM, each cell morphology is 892 represented as a cluster of square lattices identified with the identical index $\sigma$, denoting a cell; 893 the lattice distribution mainly determines an energy of the system $H$, such that cell behavior 894 depends on a balance of forces defined by the energy. The energy in our model is composed of 895 minimal factors necessary to capture the two-dimensional multicellular dynamics, such as 896 interfacial energy, cell area constraint, and active cell contraction as follows:

897

898

$$
H=\sum_{r, r^{\prime}} J_{\tau\left(\sigma_{r}\right) \tau\left(\sigma_{r^{\prime}}\right)}\left(1-\delta_{\sigma_{r} \sigma_{r^{\prime}}}\right)+\lambda_{A} \sum_{\sigma}\left(A_{\sigma}-A_{0}\right)^{2}+H_{\text {contraction }}
$$

899

900

901

902

903

904

905

906

907

908

909

910

911

912

913

914

915

916

917

918

919

920

and

921

922

$$
\Phi=\left\{\begin{array}{lll}
1+2 \omega \pi^{-1}\left(\varphi_{l_{\sigma}}-\pi\right) & \text { if } & \varphi_{l_{\sigma}}>\pi\left(1-(2 \omega)^{-1}\right) \\
0 & \text { if } & \varphi_{l_{\sigma}} \leq \pi\left(1-(2 \omega)^{-1}\right)
\end{array},\right.
$$

where each of $\boldsymbol{r}$ and $\boldsymbol{r}^{\prime}$ represents a position of lattice site, $\tau$ is an attribute of the lattice, i.e., cell or medium, $J$ is the interfacial energy between cell-cell or cell-medium, $\delta$ is the Kronecker delta, $\lambda_{A}$ is the magnitude of resistance to cell deformation, $A_{\sigma}$ is the current cell area, $A_{0}$ is the ideal cell area, and $H_{\text {contraction }}$ is a term for cell contraction.

The first term in Eq. (1) describes a strength of cell-cell adhesion. The energy to maintain cell-cell adhesion is determined by an energy of cell-cell adhesion $J_{c c}$ relative to that of cell-medium adhesion $J_{c m}$ and is expressed as $\gamma=J_{c m}-J_{c c} / 2$ (Davies and Rideal, 1963; Glazier and Graner, 1993). We determined $J_{c m}=J_{c c}=3$ in light of a balance with other parameters for imitating MDCK behaviors, and changed the value in $J_{c m}$ for the change in $\gamma$. The second term represents cell elasticity, meaning that the cells attempt to retain the ideal area. We set $\lambda_{A}$ as 0.02 and 1 when cells extend beyond and shrink below the ideal area, respectively, because of the unique material property of cells (Latorre et al., 2018; Trepat and Sahai, 2018). The ideal cell area was determined as $A_{0}=400 \mu \mathrm{m}^{2}$, by the use of actual cell images. The third term relates to the active cell contraction. In this model, individual cells are assigned unit vectors $\boldsymbol{p}_{\boldsymbol{\sigma}}$ corresponding to the front-rear cell polarity, and the cell contraction occurs depending on the polarity level $\omega$ with the contraction strength $\lambda_{\sigma}$, a variable related to the ERK activity as shall be explained later. We define the contraction term as follows:

$$
H_{\text {contraction }}=\sum_{\sigma} \sum_{l_{\sigma}} \lambda_{\sigma} \Phi
$$


924

925

926

927

928

929

930

931

932

933

934

935

936

937

938

939

940

941

942

943

944

945

946

947

948

949

950

951

952

953

954

955

956

where $l_{\sigma}$ represents the index of lattices composing the cell periphery, and $\varphi_{l_{\sigma}} \in[0, \pi]$ is an angle between $\boldsymbol{p}_{\boldsymbol{\sigma}}$ and the vector connecting from the centroid of a cell to a peripheral lattice. Note that $H_{\text {contraction }}=\sum_{\sigma} \lambda_{\sigma} P_{\sigma}$, where $P_{\sigma}$ is the perimeter of a cell when $\omega=0$, meaning that cells shrink independent of the direction of polarity. By contrast, cell contraction is biased to the rear of the cell when $\omega$ becomes larger.

In the CPM, the system transition occurs stochastically by a lattice-based Monte Carlo method; that is, the labeled value of a randomly chosen lattice site $\sigma_{r}$ is attempted to be replaced by a different labeled value of randomly-chosen adjacent lattice site $\sigma_{r}$. The transition occurs by evaluating a change in energy $\Delta H$ associated with its replacement. In the case of energy increase, i.e., $\Delta H>0$, the index replacement occurs stochastically according to a Boltzmann acceptance function $\exp (-\Delta H)$, while it deterministically occurs in the case of energy decrease, i.e., $\Delta H \leq 0$. For the details of CPM, see (Glazier and Graner, 1993; Graner and Glazier, 1992; Hirashima et al., 2017; Marée et al., 2007; Merks and Glazier, 2005; Scianna, 2015). We regard the number of trials for lattice replacement as a total number of pixel domains in simulations as a unit simulation step (USS); an update at each lattice site is attempted once per 1 USS on average. We then defined 100 USS as 1 Monte Carlo step, corresponding to 1 hour.

\section{Dynamics of polarity orientation}

Explicit rules that govern the dynamics of front-rear cell polarity have not been clear. Yet, earlier experimental studies have proposed that the front-rear polarity is oriented according to the tensile force on cell-cell junctions (Das et al., 2015; Hayer et al., 2016). In particular, it has been shown that Merlin localized at a cell-cell junction inhibits the activation of Rac1 when the tension is low. In contrast, with high tension by strong contraction of neighbor cells, Merlin is released into the cytoplasm, and Rac1 is locally activated (Das et al., 2015). Thus, orientation of the front-rear polarity in individual cells changes through physical interaction with neighboring cells over time. With this fact, we model the dynamics of cell polarity orientation $\vartheta_{\sigma}$ as a phenomenological coupling with cell displacement according to earlier theoretical studies (Hirashima et al., 2013; Notbohm et al., 2016; Peyret et al., 2019; Szabó et al., 2010a; Szabó et al., 2010b; Tlili et al., 2018):

$$
\frac{d \vartheta_{\sigma}}{d t}=\mu \frac{v_{\sigma}}{\sqrt{A_{\sigma}}} \Delta \phi_{\sigma},
$$

where $\mu$ is the degree of alignment to polarity in neighbor cells, $v_{\sigma}$ is the cell speed, and 
$957 \Delta \phi_{\sigma}$ is the angle between cell velocity $\boldsymbol{v}_{\sigma}$ and the polarity vector $\boldsymbol{p}_{\boldsymbol{\sigma}} \cdot v_{\sigma} / \sqrt{A_{\sigma}}$ contributes

958 to weighting of how much the neighboring cells affect reorientation of cell polarity and/or self-

959 reinforcement for a persistent polarization.

960 The behavior of cell polarity according to Eq. (4) can vary. For example, consider a

961 situation in which a cell A pulls an adjacent cell B in a direction opposite to the polarity

962 within cell B, and the center position of cell B is moved toward cell A. When $\mu$ is larger, the

963 polarity in cell B will re-orient to the direction in which cell B is pulled. Conversely, when $\mu$

964 is smaller, the polarity orientation in cell B will not change, and it tends to be persistent over

965 time. In addition to values of $\mu$, the magnitude of the cell displacement resulting from

966 interactions between multiple neighboring cells affects multicellular alignment of polarity. We

967 chose $\mu=1$ for reproducing a proper polarity alignment.

968

969

ERK-mediated mechanochemical feedback

970 In the main text, we have shown experimentally that cell extension activates ERK, and that the

971 ERK activation induces cell contraction. Here we incorporated this observation into the

972 framework of CPM.

973 We define the cell areal strain as $\varepsilon_{\sigma}=A_{\sigma} / A_{0}-1$, and the dynamics of a normalized

974 ERK activity level $[E R K]_{\sigma}$, bounded from -1 to 1 , is represented as

975

976

$$
\frac{d[E R K]_{\sigma}}{d t}=\left(\tanh \left(\alpha \varepsilon_{\sigma}\right)-[E R K]_{\sigma}\right) / \eta_{E}
$$

where $\alpha$ is a sensitivity parameter and $\eta_{E}$ denotes a timescale of this dynamics. We set $\alpha=$ 3 for a proper response to cell areal strains in simulations, and $\eta_{E}=3$ min with measured data in Figure 1.

$$
\frac{d \lambda_{\sigma}}{d t}=\left(\lambda U\left([E R K]_{\sigma}-[E R K]^{*}\right)-\lambda_{\sigma}\right) / \eta_{c}
$$

where $\lambda$ is a controlling parameter for conversion of ERK activation to contraction, $[E R K]^{*}$ denotes a threshold of ERK activation-induced contraction, and $\eta_{c}$ denotes a timescale of this dynamics. $U(x)$ is a step function: $U(x)=1$ for $x \geq 0$ and $U(x)=0$ for $x<0$. We 
We set $\eta_{c}=30 \mathrm{~min}$, reflecting the response time delay observed in Figure 3C.

990

\section{Simulations}

992

993

\section{Matching time}

All dynamics on polarity orientation, ERK activity, and contraction (Eq. (4)-(6)) were calculated in their discretized form and were updated every USS. The cell velocity $\boldsymbol{v}_{\sigma}$ was also calculated by the change in the center of mass of cells per 1 USS. We ran the simulation until steps equivalent to 23 hours after a start of cell migration in the confinement release assay.

Confinement release assay, Boundary conditions, and Initial conditions.

1000 In reference to a size of imaging windows, we set a pixel length of the simulation space as 2.5 $\mu \mathrm{m} \times 2.5 \mu \mathrm{m}$, and the computer simulation was performed in a space with 1200 (horizontal) $\times$ 400 (vertical) pixels, corresponding to $3 \mathrm{~mm} \times 1 \mathrm{~mm}$. We placed a cluster of cells, arranged with 120 cells in the $\mathrm{x}$-axis direction by 50 cells in the $\mathrm{y}$-axis direction, in a simulation field with a reflecting boundary condition. There is a free space only along the left side of the cell cluster, but no free space along the top, bottom, or right side. We assumed that cells facing the free space were leader cells, so that leaders could be changeable throughout the simulation. At initiation, each cell area was set to the ideal area value, and the orientation of front-rear cell polarity in each cell was random except for the leader cells. Cell proliferation was not included in simulations because it seems to minimally affect the ERK activation wave propagation (Tlili et al., 2018).

\section{Leader cells and follower cells}

1013 We defined the leader cells as cells facing a free space, corresponding to medium in the model, and follower cells as the others. The leader cells sense free spaces to migrate and communicate to the follower cells located behind the leader cells (Omelchenko et al., 2003; Yamaguchi et al., 2015). Thereby, we set the following two rules for the leader cells in polarity orientation and cell contraction. First, the cell polarity in the leader cells is persistently oriented towards the

1018 free space with a white Gaussian noise regardless of Eq. (4). Second, the leader cells keep

1019 generating polarized contractile force $(\omega=1)$ independent of cell extension-induced ERK activity $\left(\lambda_{\sigma}=100\right)$ in Eq. (2) and (3) throughout the simulations. 
1022

1023

1024

1025

1026

1027

1028

1029

1030

1031

1032

1033

1034

1035

1036

1037

1038

1039

1040

1041

1042

1043

1044

1045

1046

1047

1048

1049

1050

1051

1052

1053

1054

\section{Parameters}

The default parameter values are shown in the column "Values" in Table S1. In the rightmost column, the range of the respective parameters used in Figure 7 is summarized (Table S1). Each parameter value was determined based on either measured data, observation, or a balance of other parameter values. Under an energy minimization framework, relative values of a parameter to values in other parameters should be significant if the system is not far from its equilibrium.

\section{Additional comments on modeling and simulations}

Wave propagation of cell velocities and/or mechanical stress during collective cell migration have been modeled in some earlier studies, and most of them adopt a continuum approach (Alert and Trepat, 2019; Banerjee et al., 2015; Notbohm et al., 2016; Tlili et al., 2018; Yabunaka and Marcq, 2017). They are constructed with a relatively simple assumption and include just a few parameters, which makes the analysis easier. In contrast, we chose a cell-based approach to express the collective cell behavior and modeled a system on a cellular mechanochemical feedback at tissue scale. Despite being complex and limited to numerical simulations, our model can recapitulate multiple unidirectional ERK activation waves in collective cell migration. Moreover, it simulates conditions under which the waves are not generated, and those are consistent with our experimental results. We believe that this approach contributes to further understanding of unique systems within and between cells, as well as refining our theoretical modeling of such systems. Additional numerical investigations on multicellular dynamics and ERK activation waves were beyond the scope of this study.

\section{Repeatability of experiments}

All experiments were performed on at least three independent cell culture preparations.

\section{QUANTIFICATION AND STATISTICAL ANALYSIS}

\section{Quantification of ERK activity and cell deformation}

For the analysis of the cell strain rate along an axis of collective cell migration ( $\mathrm{x}$-strain rate), PIV using MatPIV (Sveen, 2004) was applied to phase contrast time-lapse images to calculate velocity fields of cells. Velocity fields at time $T$ were computed by the displacement between the images at $T-\Delta t$ and $T+\Delta t$ by using 'single' option for the method of pattern matching. The 
1055

1056

1057

1058

1059

1060

1061

1062

1063

1064

1065

1066

1067

1068

1069

1070

1071

1072

1073

1074

1075

1076

1077

1078

1079

1080

1081

1082

1083

size of the interrogation window was set to $29.1 \mu \mathrm{m}$, approximately corresponding to the typical cell size, and the window overlap was set to $50 \%$. Here, we defined the $\mathrm{x}$-strain rate as spatial derivative of velocity field along $\mathrm{x}$-axis divided by the distance between the centers of two adjacent interrogation windows. Thus, the x-strain rate $\dot{\varepsilon}_{i, j}$ was calculated according to

$$
\dot{\varepsilon}_{i, j}=\frac{v_{i, j}^{x}-v_{i+1, j}^{x}}{L}
$$

where $v_{i, j}^{x}$ is the $x$ component of the velocity at spatial indices of resultant velocity vectors obtained by PIV analysis $(i, j)$, and $L$ is the distance between the windows $(14.5 \mu \mathrm{m})$. The value of $\mathrm{x}$-strain rate is assigned at the center between the $\mathrm{x}$-coordinate of two source velocity vectors. The obtained discrete data were processed by a cubic interpolation method to fill the gap.

To represent the FRET efficiency, FRET/CFP ratio images were generated after the background intensity was subtracted from the original fluorescence images in the CFP and FRET channel, respectively, using Metamorph software (Molecular Devices, Sunnyvale, CA). Then, the Fiji TrackMate plugin was applied to the CFP fluorescence images for tracking each cell. The time derivative of ERK activity (ERK activation rate) at time $t$ for each cell was computed by the change in FRET/CFP ratio between $t-\Delta t$ and $t+\Delta t$. The obtained time-series data of the $\mathrm{x}$-strain rate and the ERK activation rate were processed with a Savitzky-Golay filter to reduce the noise. The cross-correlation coefficient $r(\tau)$ between time-series data of $\mathrm{X}$-strain rate $f(t)$ and ERK activation rate $g(t)$ was calculated as follows:

$$
r(\tau)=\frac{\sum_{t} f(t+\tau) g(t)}{\sqrt{\sum_{t} f(t)^{2}} \sqrt{\sum_{t} g(t)^{2}}},
$$

where $\tau$ is the lag time.

To quantify the amplitude of $\mathrm{x}$-strain rate oscillation, the time-series data were fitted to a multi-peak function as described previously (Muta et al., 2018).

\section{Quantification of directedness}

For quantification of directionality of Golgi positioning, ERK activation waves, and cell migration, we defined directedness as shown in Figure 5D. The reference direction is set to the forward direction for analysis of Golgi orientation and cell migration direction, and to the backward direction for that of ERK wave direction to match the signs of their directedness. To obtain the sample directions of the Golgi apparatus, the center of mass of the nucleus in each cell was determined with Fiji. Fluorescence images of Golgi apparatus markers were processed with a mean filter. The position that showed the highest intensity in the region within $6.8 \mu \mathrm{m}$ 
1084 from the center of mass of the nucleus was defined as the position of the Golgi apparatus. The

1085 sample directions were determined by the direction of the Golgi apparatus relative to the center

1086 of mass of the nucleus. To determine the sample directions of ERK activation waves, heat maps

1087 of ERK activity were obtained by interpolating the signals in regions between the nuclei of

$1088 \mathrm{MDCK} /$ EKAREV-NLS cells in the FRET/CFP ratio images. The heat maps of ERK activity 1089 were analyzed by MatPIV with a $174 \mu \mathrm{m}$ window size and a 75\% window overlap. The 1090 directions of the calculated velocity vectors were obtained as the sample directions. For the 1091 determination of the sample directions of cell migration, each cell was tracked with a Fiji 1092 TrackMate plugin. The direction of cell displacement for $20 \mathrm{~min}$ was defined as the sample 1093 direction of cell migration for that individual cell.

\section{Statistical analysis}

1096 Statistical analyses were performed with GraphPad Prism 7 software (GraphPad Software, San

1097 Diego, CA). No statistical analysis was used to predetermine the sample size. The sample sizes, 1098 statistical tests, and p-values are indicated in the figures and the figure legends. To compare two 1099 sets of data, paired or unpaired $t$-tests were used. To compare multiple sets of data, the samples 1100 were analyzed by one-way ANOVA followed by Dunnett's multiple comparison test. P-values 1101 of less than 0.05 were considered to be statistically significant in two-tailed tests, and were 1102 classified as follows: ${ }^{*} \mathrm{P}<0.05,{ }^{* *} \mathrm{P}<0.01,{ }^{* * *} \mathrm{P}<0.001,{ }^{* * * *} \mathrm{P}<0.0001$, and n.s. (not significant, i.e., $\mathrm{P} \geq 0.05$ ).

1104 
1106

1107

1108

1109

1110

1111

1112

1113

1114

1115

1116

1117

1118

1119

1120

1121

1122

1123

1124

1125

1126

1127

1128

1129

1130

1131

1132

1133

1134

1135

1136

1137

1138

Supplemental video titles and legends

Movie S1. ERK activation waves and cell deformation waves during collective cell migration, related to Figure 1

Time-lapse video of collectively migrating MDCK cells expressing EKAREV-NLS. Phase contrast images are shown at the top. The golden pseudo-color represents the FRET/CFP ratio indicating ERK activity (middle). Red and blue indicate positive and negative $\mathrm{x}$-strain rate, respectively (bottom). The color scales correspond to those in Figure 1A. Time in hr:min.

\section{Movie S2. Cell contraction upon optogenetic ERK activation, related to Figure 3}

Time-lapse video of the boundary between confluent MDCK cells with and without 2paRAF expression. The upper frames represent the fluorescence of CIBN-mScarlet-I-CAAX, a component of $2 \mathrm{paRAF}$. The lower images show differential interference contrast (DIC).

The blue light illumination started at $0 \mathrm{~min}$ and was repeated every $5 \mathrm{~min}$. The cells were treated with DMSO (left), $200 \mathrm{nM}$ trametinib (center), $10 \mu \mathrm{M}$ Y-27632 (right) $30 \mathrm{~min}$ before the start of the imaging. Time in hr:min.

Movie S3. ROCK activity propagation during collective cell migration, related to Figure 3

First part: Time-lapse video of collectively migrating MDCK cells expressing a FRET biosensor for ROCK activity. The color represents the FRET/CFP ratio indicating ROCK activity and its scale corresponds to the one in Figure 3F. Second part shows migrating cells that were treated with $200 \mathrm{nM}$ trametinib (left) and $100 \mu \mathrm{M}$ Y-27632 (right) at 0 min. Time in hr:min.

\section{Movie S4. Traction force microscopy with optogenetic ERK activation, related to Figure} 3

Time-lapse traction force microscopy at the boundary between confluent MDCK cells with and without 2paRAF expression. Upper frames show differential interference contrast (DIC). Middle frames represent the fluorescence of CIBN-mScarlet-I-CAAX, a component of 2paRAF. Lower frames show traction forces. The color scale corresponds to the one in Figure 3L. The blue light illumination started at $0 \mathrm{~min}$ and was repeated every $5 \mathrm{~min}$. Time in hr:min.

\section{Movie S5. Cell contraction triggers ERK activation waves, related to Figure 4}

Time-lapse videos of the boundary between confluent MDCK cells with and without 
1139 rapamycin-activatable Rho GEF (RA Rho GEF). The approximate boundary is indicated as 1140 dotted lines at the initial time frame. The color represents the FRET/CFP ratio indicating ERK 1141 activity, and the scale corresponds to the one in Figure 4B. The cells were treated with DMSO 1142 (left) or $50 \mu \mathrm{M}$ rapamycin (right) at 0 min to induce contraction of the RA Rho GEF-expressing 1143 cells. Time in hr:min.

Movie S6. Intercellular mechanical linkage is required for ERK activation waves, related to Figure 4

1147 Time-lapse videos of collectively migrating WT (left) and $\alpha$-catenin KO (right) MDCK cells.

1148 The color represents the FRET/CFP ratio indicating ERK activity, as shown in Figure 4, and its upper and lower value is 1.8 and 0.85 . Time in $\mathrm{hr}: \mathrm{min}$.

Movie S7. Requirement of Cdc42 and Rac1 for unidirectional ERK activation waves, related to Figure 5

1153 Time-lapse videos of collectively migrating WT (upper), shCdc42 (middle), and shRac1 1154 (lower)-expressing MDCK cells. The color represents the FRET/CFP ratio indicating ERK activity, as shown in Figure 1, and its upper and lower value is 1.8 and 0.85 for each of the videos. Time in hr:min.

Movie S8. Effect of constitutive ERK activation and inhibition on collective cell migration, related to Figure 6

1160 Time-lapse videos of collectively migrating MDCK cells. The color represents the FRET/CFP

1161 ratio indicating ERK activity, and the scale corresponds to the one in Figure S4D and S4E. The 1162 cells were treated with DMSO (upper), $200 \mathrm{nM}$ trametinib (middle), and $10 \mathrm{nM}$ TPA (bottom) 1163 at $0 \mathrm{~min}$. Time in hr:min.

Movie S9. Optogenetic ERK activation waves and front-rear cell polarization, related to

\section{Figure 6}

1167 Time-lapse videos of migrating MDCK cells without (upper) and with (lower) Optogenetic

1168 ERK activation waves. Green and red indicate nuclei (EKAREV-NLS) and Golgi apparatus 1169 (Golgi-7-mCherry), respectively. Cyan represents regions illuminated with blue light. The cells 1170 were pre-treated with $1 \mu \mathrm{M}$ PD153035, an EGFR inhibitor, to suppress autonomous ERK 1171 activation waves. Time in hr:min. 
1172

1173 Movie S10. in silico ERK activation waves, related to Figure 7

1174 Simulations for ERK activation waves in migrating cells with ( $\omega=1$, upper frame) and without

$1175(\omega=0$, lower frame $)$ cell polarity. The color represents the ERK activity and its scale corresponds 1176 to the one in Figure 7C.

1177 
1178

1179

1180

1181

1182

1183

1184

1185

1186

1187

1188

1189

1190

1191

1192

1193

1194

1195

1196

1197

1198

1199

1200

1201

1202

1203

1204

1205

\section{References}

Acharya, B.R., Nestor-Bergmann, A., Liang, X., Gupta, S., Duszyc, K., Gauquelin, E., Gomez, G.A., Budnar, S., Marcq, P., Jensen, O.E., et al. (2018). A Mechanosensitive RhoA Pathway that Protects Epithelia against Acute Tensile Stress. Dev Cell 47, 439-452 e436.

Akagi, T., Sasai, K., and Hanafusa, H. (2003). Refractory nature of normal human diploid fibroblasts with respect to oncogene-mediated transformation. P Natl Acad Sci USA 100, 13567-13572.

Amano, M., Ito, M., Kimura, K., Fukata, Y., Chihara, K., Nakano, T., Matsuura, Y., and Kaibuchi, K. (1996). Phosphorylation and activation of myosin by Rho-associated kinase (Rho-kinase). Journal of Biological Chemistry 271, 20246-20249.

Aoki, K., Kondo, Y., Naoki, H., Hiratsuka, T., Itoh, R.E., and Matsuda, M. (2017).

Propagating Wave of ERK Activation Orients Collective Cell Migration. Dev Cell 43, 305317 e305.

Aoki, K., Kumagai, Y., Sakurai, A., Komatsu, N., Fujita, Y., Shionyu, C., and Matsuda, M. (2013). Stochastic ERK activation induced by noise and cell-to-cell propagation regulates cell density-dependent proliferation. Mol Cell 52, 529-540.

Aoki, K., and Matsuda, M. (2009). Visualization of small GTPase activity with fluorescence resonance energy transfer-based biosensors. Nature Protocols 4, 1623-1631.

Carvalho, J.R., Fortunato, I.C., Fonseca, C.G., Pezzarossa, A., Barbacena, P., DominguezCejudo, M.A., Vasconcelos, F.F., Santos, N.C., Carvalho, F.A., and Franco, C.A. (2019). Noncanonical Wnt signaling regulates junctional mechanocoupling during angiogenic collective cell migration. Elife 8 .

Colo, G.P., Hernandez-Varas, P., Lock, J., Bartolome, R.A., Arellano-Sanchez, N., Stromblad, S., and Teixido, J. (2012). Focal adhesion disassembly is regulated by a RIAM to MEK-1 pathway. J Cell Sci 125, 5338-5352.

Das, T., Safferling, K., Rausch, S., Grabe, N., Boehm, H., and Spatz, J.P. (2015). A molecular mechanotransduction pathway regulates collective migration of epithelial cells. Nat Cell Biol $17,276-287$. 
1206 Diaz-Rodriguez, E., Montero, J.C., Esparis-Ogando, A., Yuste, L., and Pandiella, A. (2002).

1207 Extracellular signal-regulated kinase phosphorylates tumor necrosis factor alpha-converting 1208 enzyme at threonine 735: a potential role in regulated shedding. Mol Biol Cell 13, 2031-2044.

1209 Farooqui, R., and Fenteany, G. (2005). Multiple rows of cells behind an epithelial wound edge 1210 extend cryptic lamellipodia to collectively drive cell-sheet movement. J Cell Sci 118, 51-63.

1211 Friedl, P., and Gilmour, D. (2009). Collective cell migration in morphogenesis, regeneration 1212 and cancer. Nat Rev Mol Cell Biol 10, 445-457.

1213 Fujioka, A., Terai, K., Itoh, R.E., Aoki, K., Nakamura, T., Kuroda, S., Nishida, E., and 1214 Matsuda, M. (2006). Dynamics of the Ras/ERK MAPK cascade as monitored by fluorescent 1215 probes. J Biol Chem 281, 8917-8926.

1216 Glazier, J.A., and Graner, F. (1993). Simulation of the differential adhesion driven 1217 rearrangement of biological cells. Phys Rev E Stat Phys Plasmas Fluids Relat Interdiscip 1218 Topics 47, 2128-2154.

1219 Gudipaty, S.A., Lindblom, J., Loftus, P.D., Redd, M.J., Edes, K., Davey, C.F., Krishnegowda, 1220 V., and Rosenblatt, J. (2017). Mechanical stretch triggers rapid epithelial cell division through 1221 Piezo1. Nature 543, 118-121.

1222 Guillot, C., and Lecuit, T. (2013). Mechanics of epithelial tissue homeostasis and 1223 morphogenesis. Science 340, 1185-1189.

1224 Guilluy, C., Garcia-Mata, R., and Burridge, K. (2011). Rho protein crosstalk: another social 1225 network? Trends Cell Biol 21, 718-726.

1226 Haeger, A., Wolf, K., Zegers, M.M., and Friedl, P. (2015). Collective cell migration: guidance 1227 principles and hierarchies. Trends Cell Biol 25, 556-566.

1228 Handly, L.N., Pilko, A., and Wollman, R. (2015). Paracrine communication maximizes 1229 cellular response fidelity in wound signaling. Elife 4, e09652.

1230 Harvey, C.D., Ehrhardt, A.G., Cellurale, C., Zhong, H., Yasuda, R., Davis, R.J., and Svoboda, 1231 K. (2008). A genetically encoded fluorescent sensor of ERK activity. Proc Natl Acad Sci U S 1232 A $105,19264-19269$. 
1233 Heisenberg, C.P., and Bellaiche, Y. (2013). Forces in tissue morphogenesis and patterning. 1234 Cell 153, 948-962.

1235 Hirashima, T., Hosokawa, Y., Iino, T., and Nagayama, M. (2013). On fundamental cellular 1236 processes for emergence of collective epithelial movement. Biol Open 2, 660-666.

1237 Hirashima, T., Rens, E.G., and Merks, R.M.H. (2017). Cellular Potts modeling of complex 1238 multicellular behaviors in tissue morphogenesis. Dev Growth Differ 59, 329-339.

1239 Hoffmann, H. (2020). Violin Plot. MATLAB Central File Exchange.

1240 Inoue, T., Heo, W.D., Grimley, J.S., Wandless, T.J., and Meyer, T. (2005). An inducible 1241 translocation strategy to rapidly activate and inhibit small GTPase signaling pathways. Nat 1242 Methods 2, 415-418.

1243 Jaqaman, K., Loerke, D., Mettlen, M., Kuwata, H., Grinstein, S., Schmid, S.L., and Danuser, 1244 G. (2008). Robust single-particle tracking in live-cell time-lapse sequences. Nat Methods 5, $1245 \quad 695-702$.

1246 Kawabata, N., and Matsuda, M. (2016). Cell Density-Dependent Increase in Tyrosine1247 Monophosphorylated ERK2 in MDCK Cells Expressing Active Ras or Raf. PLoS One 11, $1248 \mathrm{e} 0167940$.

1249 Kennedy, M.J., Hughes, R.M., Peteya, L.A., Schwartz, J.W., Ehlers, M.D., and Tucker, C.L. 1250 (2010). Rapid blue-light-mediated induction of protein interactions in living cells. Nature 1251 Methods 7, 973-U948.

1252 Kimura, K., Ito, M., Amano, M., Chihara, K., Fukata, Y., Nakafuku, M., Yamamori, B., Feng, 1253 J.H., Nakano, T., Okawa, K., et al. (1996). Regulation of myosin phosphatase by Rho and 1254 Rho-Associated kinase (Rho-kinase). Science 273, 245-248.

1255 Kinjo, T., Terai, K., Horita, S., Nomura, N., Sumiyama, K., Togashi, K., Iwata, S., and 1256 Matsuda, M. (2019). FRET-assisted photoactivation of flavoproteins for in vivo two-photon 1257 optogenetics. Nat Methods 16, 1029-1036.

1258 Kippenberger, S., Loitsch, S., Guschel, M., Muller, J., Knies, Y., Kaufmann, R., and Bernd, A. 1259 (2005). Mechanical stretch stimulates protein kinase B/Akt phosphorylation in epidermal cells 1260 via angiotensin II type 1 receptor and epidermal growth factor receptor. J Biol Chem 280, 
1261

1262

1263

1264

1265

1266

1267

1268

1269

1270

1271

1272

1273

1274

1275

1276

1277

1278

1279

1280

1281

1282

1283

1284

1285

1286

1287

3060-3067.

Komatsu, N., Aoki, K., Yamada, M., Yukinaga, H., Fujita, Y., Kamioka, Y., and Matsuda, M. (2011). Development of an optimized backbone of FRET biosensors for kinases and GTPases. Mol Biol Cell 22, 4647-4656.

Li, C., Imanishi, A., Komatsu, N., Terai, K., Amano, M., Kaibuchi, K., and Matsuda, M. (2017). A FRET Biosensor for ROCK Based on a Consensus Substrate Sequence Identified by KISS Technology. Cell Struct Funct 42, 1-13.

Li, R., Wang, T., Walia, K., Gao, B., and Krepinsky, J.C. (2018). Regulation of profibrotic responses by ADAM17 activation in high glucose requires its C-terminus and FAK. J Cell Sci 131.

Magdalena, J., Millard, T.H., and Machesky, L.M. (2003). Microtubule involvement in NIH 3T3 Golgi and MTOC polarity establishment. J Cell Sci 116, 743-756.

Maretzky, T., Evers, A., Zhou, W., Swendeman, S.L., Wong, P.M., Rafii, S., Reiss, K., and Blobel, C.P. (2011a). Migration of growth factor-stimulated epithelial and endothelial cells depends on EGFR transactivation by ADAM17. Nat Commun 2, 229.

Maretzky, T., Zhou, W., Huang, X.Y., and Blobel, C.P. (2011b). A transforming Src mutant increases the bioavailability of EGFR ligands via stimulation of the cell-surface metalloproteinase ADAM17. Oncogene 30, 611-618.

Matsubayashi, Y., Ebisuya, M., Honjoh, S., and Nishida, E. (2004). ERK activation propagates in epithelial cell sheets and regulates their migration during wound healing. Curr Biol 14, 731-735.

Mayor, R., and Etienne-Manneville, S. (2016). The front and rear of collective cell migration. Nat Rev Mol Cell Biol 17, 97-109.

Merks, R.M.H., and Glazier, J.A. (2005). A cell-centered approach to developmental biology. Physica A: Statistical Mechanics and its Applications 352, 113-130.

Miyoshi, H., Blomer, U., Takahashi, M., Gage, F.H., and Verma, I.M. (1998). Development of a self-inactivating lentivirus vector. J Virol 72, 8150-8157. 
1288 Moreno, E., Valon, L., Levillayer, F., and Levayer, R. (2019). Competition for Space Induces 1289 Cell Elimination through Compaction-Driven ERK Downregulation. Curr Biol 29, 23-34 e28.

1290 Muta, Y., Fujita, Y., Sumiyama, K., Sakurai, A., Taketo, M.M., Chiba, T., Seno, H., Aoki, K., 1291 Matsuda, M., and Imajo, M. (2018). Composite regulation of ERK activity dynamics 1292 underlying tumour-specific traits in the intestine. Nat Commun 9, 2174.

1293 Nagafuchi, A., Ishihara, S., and Tsukita, S. (1994). The roles of catenins in the cadherin1294 mediated cell adhesion: functional analysis of E-cadherin-alpha catenin fusion molecules. J 1295 Cell Biol 127, 235-245.

1296 Naito, Y., Hino, K., Bono, H., and Ui-Tei, K. (2015). CRISPRdirect: software for designing 1297 CRISPR/Cas guide RNA with reduced off-target sites. Bioinformatics (Oxford, England) 31, 1298 1120-1123.

1299 Nikolic, D.L., Boettiger, A.N., Bar-Sagi, D., Carbeck, J.D., and Shvartsman, S.Y. (2006). Role 1300 of boundary conditions in an experimental model of epithelial wound healing. American 1301 journal of physiology Cell physiology 291, C68-75.

1302 Niu, A., Wang, B., and Li, Y.P. (2015). TNFalpha shedding in mechanically stressed 1303 cardiomyocytes is mediated by Src activation of TACE. J Cell Biochem 116, 559-565.

1304 Nobes, C.D., and Hall, A. (1999). Rho GTPases control polarity, protrusion, and adhesion 1305 during cell movement. J Cell Biol 144, 1235-1244.

1306 Omelchenko, T., Vasiliev, J.M., Gelfand, I.M., Feder, H.H., and Bonder, E.M. (2003). Rho1307 dependent formation of epithelial "leader" cells during wound healing. P Natl Acad Sci USA 1308 100, 10788-10793.

1309 Petitjean, L., Reffay, M., Grasland-Mongrain, E., Poujade, M., Ladoux, B., Buguin, A., and 1310 Silberzan, P. (2010). Velocity fields in a collectively migrating epithelium. Biophys J 98, $1311 \quad 1790-1800$.

1312 Peyret, G., Mueller, R., d'Alessandro, J., Begnaud, S., Marcq, P., Mege, R.M., Yeomans, J.M., 1313 Doostmohammadi, A., and Ladoux, B. (2019). Sustained Oscillations of Epithelial Cell 1314 Sheets. Biophys J 117, 464-478.

1315 Reffay, M., Parrini, M.C., Cochet-Escartin, O., Ladoux, B., Buguin, A., Coscoy, S., Amblard, 
1316 F., Camonis, J., and Silberzan, P. (2014). Interplay of RhoA and mechanical forces in

1317 collective cell migration driven by leader cells. Nat Cell Biol 16, 217-223.

1318 Reffay, M., Petitjean, L., Coscoy, S., Grasland-Mongrain, E., Amblard, F., Buguin, A., and 1319 Silberzan, P. (2011). Orientation and polarity in collectively migrating cell structures: statics 1320 and dynamics. Biophys J 100, 2566-2575.

1321 Rodriguez-Franco, P., Brugues, A., Marin-Llaurado, A., Conte, V., Solanas, G., Batlle, E., 1322 Fredberg, J.J., Roca-Cusachs, P., Sunyer, R., and Trepat, X. (2017). Long-lived force patterns and deformation waves at repulsive epithelial boundaries. Nat Mater 16, 1029-1037.

Sahin, U., Weskamp, G., Kelly, K., Zhou, H.M., Higashiyama, S., Peschon, J., Hartmann, D., Saftig, P., and Blobel, C.P. (2004). Distinct roles for ADAM10 and ADAM17 in ectodomain shedding of six EGFR ligands. J Cell Biol 164, 769-779.

1327 Sakurai, A., Matsuda, M., and Kiyokawa, E. (2012). Activated Ras protein accelerates cell 1328 cycle progression to perturb Madin-Darby canine kidney cystogenesis. J Biol Chem 287, $132931703-31711$.

1330 Schindelin, J., Arganda-Carreras, I., Frise, E., Kaynig, V., Longair, M., Pietzsch, T., Preibisch, 1331 S., Rueden, C., Saalfeld, S., Schmid, B., et al. (2012). Fiji: an open-source platform for 1332 biological-image analysis. Nat Methods 9, 676-682.

1333 Serra-Picamal, X., Conte, V., Vincent, R., Anon, E., Tambe, D.T., Bazellieres, E., Butler, J.P., 1334 Fredberg, J.J., and Trepat, X. (2012). Mechanical waves during tissue expansion. Nature 1335 Physics 8, 628-634.

1336 Shellard, A., and Mayor, R. (2019). Supracellular migration - beyond collective cell 1337 migration. J Cell Sci 132.

1338 Sveen, J. K. (2004). An introduction to MatPIV v. 1.6.1. eprint series, Dept. of Math. 1339 University of Oslo

1340 Tambe, D.T., Hardin, C.C., Angelini, T.E., Rajendran, K., Park, C.Y., Serra-Picamal, X., 1341 Zhou, E.H., Zaman, M.H., Butler, J.P., Weitz, D.A., et al. (2011). Collective cell guidance by 1342 cooperative intercellular forces. Nat Mater 10, 469-475.

1343 Tanabe, Y., Koga, M., Saito, M., Matsunaga, Y., and Nakayama, K. (2004). Inhibition of 
1344 adipocyte differentiation by mechanical stretching through ERK-mediated downregulation of

1345 PPARgamma2. J Cell Sci 117, 3605-3614.

1346 Tlili, S., Gauquelin, E., Li, B., Cardoso, O., Ladoux, B., Delanoe-Ayari, H., and Graner, F.

1347 (2018). Collective cell migration without proliferation: density determines cell velocity and

1348 wave velocity. R Soc Open Sci 5, 172421.

1349 Totsukawa, G., Yamakita, Y., Yamashiro, S., Hartshorne, D.J., Sasaki, Y., and Matsumura, F.

1350 (2000). Distinct roles of ROCK (Rho-kinase) and MLCK in spatial regulation of MLC

1351 phosphorylation for assembly of stress fibers and focal adhesions in 3T3 fibroblasts. J Cell

1352 Biol 150, 797-806.

1353 Trepat, X., Wasserman, M.R., Angelini, T.E., Millet, E., Weitz, D.A., Butler, J.P., and

1354 Fredberg, J.J. (2009). Physical forces during collective cell migration. Nature Physics 5, 4261355430.

1356 Urasaki, A., Morvan, G., and Kawakami, K. (2006). Functional dissection of the Tol2

1357 transposable element identified the minimal cis-sequence and a highly repetitive sequence in

1358 the subterminal region essential for transposition. Genetics 174, 639-649.

1359 van Unen, J., Reinhard, N.R., Yin, T., Wu, Y.I., Postma, M., Gadella, T.W., and Goedhart, J.

1360 (2015). Plasma membrane restricted RhoGEF activity is sufficient for RhoA-mediated actin 1361 polymerization. Sci Rep 5, 14693.

1362 Webb, D.J., Donais, K., Whitmore, L.A., Thomas, S.M., Turner, C.E., Parsons, J.T., and 1363 Horwitz, A.F. (2004). FAK-Src signalling through paxillin, ERK and MLCK regulates 1364 adhesion disassembly. Nat Cell Biol 6, 154-161.

1365 Yamaguchi, N., Mizutani, T., Kawabata, K., and Haga, H. (2015). Leader cells regulate 1366 collective cell migration via Rac activation in the downstream signaling of integrin beta 1 and 1367 PI3K. Sci Rep 5, 7656.

1368 Zhang, B., Peng, F., Wu, D., Ingram, A.J., Gao, B., and Krepinsky, J.C. (2007). Caveolin-1 1369 phosphorylation is required for stretch-induced EGFR and Akt activation in mesangial cells. 1370 Cell Signal 19, 1690-1700.

1372 Alert, R. and Trepat, X. (2019). Physical Models of Collective Cell Migration. 
1373

1374

1375

1376

1377

1378

1379

1380

1381

1382

1383

1384

1385

1386

1387

1388

1389

1390

1391

1392

1393

1394

1395

1396

1397

1398

1399

1400

1401

1402

1403

1404

1405

Balter, A., Merks, R. M. H., Poplawski, N. J., Swat, M. and Glazier, J. a (2007). The Glazier-Graner-Hogeweg Model: Extensions, Future Directions, and Opportunities for Further Study. Single-Cell-Based Model. Biol. Med. 2, 17.

Banerjee, S., Utuje, K. J. C. and Marchetti, M. C. (2015). Propagating Stress Waves during Epithelial Expansion. Phys. Rev. Lett.

Das, T., Safferling, K., Rausch, S., Grabe, N., Boehm, H. and Spatz, J. P. (2015). A molecular mechanotransduction pathway regulates collective migration of epithelial cells. Nat. Cell Biol. 17, 276-287.

Davies, J. T. and Rideal, E. K. (1963). Interfacial phenomena.

Glazier, J. A. and Graner, F. (1993). Simulation of the differential adhesion driven rearrangement of biological cells. Phys. Rev. E 47, 2128-2154.

Graner, F. and Glazier, J. A. (1992). Simulation of Biological Cell Sorting Using TwoDimensiona Extended Potts Model. Phys. Rev. Lett. 69, 2013-2016.

Hayer, A., Shao, L., Chung, M., Joubert, L. M., Yang, H. W., Tsai, F. C., Bisaria, A., Betzig, E. and Meyer, T. (2016). Engulfed cadherin fingers are polarized junctional structures between collectively migrating endothelial cells. Nat. Cell Biol. 18, 13111323.

Hirashima, T., Hosokawa, Y., Iino, T. and Nagayama, M. (2013). On fundamental cellular processes for emergence of collective epithelial movement. Biol. Open 2, 660-6.

Hirashima, T., Rens, E. G. and Merks, R. M. H. (2017). Cellular Potts modeling of complex multicellular behaviors in tissue morphogenesis. Dev. Growth Differ. 59,.

Krieg, M., Arboleda-Estudillo, Y., Puech, P.-H., Käfer, J., Graner, F., Müller, D. J. and Heisenberg, C.-P. C.-P. (2008). Tensile forces govern germ-layer organization in zebrafish. Nat. Cell Biol. 10, 429-36.

Latorre, E., Kale, S., Casares, L., Gómez-González, M., Uroz, M., Valon, L., Nair, R. V, Garreta, E., Montserrat, N., del Campo, A., et al. (2018). Active superelasticity in three-dimensional epithelia of controlled shape. Nature 563, 203-208.

Marée, A. F. M., Grieneisen, V. a and Hogeweg, P. (2007). The Cellular Potts Model and Biophysical Properties of Cells, Tissues and Morphogenesis. Single-Cell-Based Model. Biol. Med. 2, 30.

Merks, R. M. H. and Glazier, J. A. (2005). A cell-centered approach to developmental biology. Phys. A Stat. Mech. its Appl. 352, 113-130.

Notbohm, J., Banerjee, S., Utuje, K. J. C., Gweon, B., Jang, H., Park, Y., Shin, J., Butler, 
1406

1407

1408

1409

1410

1411

1412

1413

1414

1415

1416

1417

1418

1419

1420

1421

1422

1423

1424

1425

1426

1427

1428

1429

1430

1431

1432

1433

1434

1435

J. P., Fredberg, J. J. and Marchetti, M. C. (2016). Cellular Contraction and Polarization Drive Collective Cellular Motion. Biophys. J. 110, 2729-2738.

Omelchenko, T., Vasiliev, J. M., Gelfand, I. M., Feder, H. H. and Bonder, E. M. (2003). Rho-dependent formation of epithelial "leader" cells during wound healing. Proc. Natl. Acad. Sci.

Peyret, G., Mueller, R., D’Alessandro, J., Begnaud, S., Marcq, P., Mège, R.-M., Yeomans, J. M., Doostmohammadi, A. and Ladoux, B. (2019). Sustained Oscillations of Epithelial Cell Sheets. Biophys. J. 117, 464-478.

Scianna, M. (2015). An extended Cellular Potts Model analyzing a wound healing assay. Comput. Biol. Med. 62, 33-54.

Szabó, A., Ünnep, R., Méhes, E., Twal, W. O., Argraves, W. S., Cao, Y. and Czirók, A. (2010a). Collective cell motion in endothelial monolayers. Phys. Biol. 7,

Szabó, A., Ünnep, R., Méhes, E., Twal, W. O., Argraves, W. S., Cao, Y., Czirók, A., Unnep, R., Méhes, E., Twal, W. O., et al. (2010b). Collective cell motion in endothelial monolayers. Phys. Biol. 7, 046007.

te Boekhorst, V., Preziosi, L. and Friedl, P. (2016). Plasticity of Cell Migration In Vivo and In Silico. Annu. Rev. Cell Dev. Biol. 32, 491-526.

Tlili, S., Gauquelin, E., Li, B., Cardoso, O., Ladoux, B., Ayari, H. D. and Graner, F. (2018). Collective cell migration without proliferation: Density determines cell velocity and wave velocity. R. Soc. Open Sci.

Trepat, X. and Sahai, E. (2018). Mesoscale physical principles of collective cell organization. Nat. Phys.

van Helvert, S., Storm, C. and Friedl, P. (2018). Mechanoreciprocity in cell migration. Nat. Cell Biol. 20, 8-20.

Yabunaka, S. and Marcq, P. (2017). Emergence of epithelial cell density waves. Soft Matter.

Yamaguchi, N., Mizutani, T., Kawabata, K. and Haga, H. (2015). Leader cells regulate collective cell migration via Rac activation in the downstream signaling of integrin $\beta 1$ and PI3K. Sci. Rep. 